\title{
ECOTOXICITY OF SELECTED PHARMACEUTICALS OF URBAN ORIGIN DISCHARGED TO THE SAINT-LAWRENCE RIVER (QUÉBEC, CANADA): A REVIEW
}

\author{
BLAISE'1, C.; GAGNÉ1', F.; EULLAFFROY², P. \& FÉRARD³, J-F. \\ 1Environment Canada, 105 McGill Street, Montréal, Québec \\ Canada, H2Y 2E7. christian.blaise@ec.gc.ca
}

2Laboratoire Plantes, Pesticides et Développement Durable, EA 2069, (URVVC), BP 1039 Université de Reims Champagne-Ardenne, 51687 Reims Cedex 2, France ${ }^{3}$ Laboratoire Ecotoxicité et Santé Environnementale, Université Paul Verlaine, CNRS FRE 2635,
Campus Bridoux, rue du Général Delestraint, 57070 Metz, France

\begin{abstract}
Blaise, C.; Gagné, F.; Eullaffroy, P. \& Férard, J-F. 2006. Ecotoxicity of selected pharmaceuticals of urban origin discharged to the Saint-Lawrence River (Québec, Canada) : a review. Braz. J. Aquat. Sci. Technol. 10(2):29-51. ISSN 1808-7035. Twelve pharmaceuticals comprising anti-inflammatory agents (ibuprofen, naproxen), lipid regulators (bezafibrate, gemfibrozil), an anti-convulsant (carbamazepine), antibiotics (sulfamethoxazole, sulfapyridine, oxytetracycline, novobiocin, trimethoprim), a stimulant (caffeine) and a nicotine metabolite (cotinine) were identified in the final effluent of a major wastewater treatment plant (WWTP) based in the Montreal area (Québec, Canada) discharging to the Saint-Lawrence River. Their measured concentrations ranged from 33 (carbamazepine) to $22187 \mathrm{ng} / \mathrm{L}$ (caffeine) in the Montreal WWTP effluent and from 58 (cotinine) to 85000 (ibuprofen) $\mathrm{ng} / \mathrm{L}$ based on maximum concentrations found in other municipal effluents as reported elsewhere. In general, elimination of these drugs by wastewater treatment is purported to be fairly efficient for ibuprofen, naproxen, bezafibrate, gemfibrozil and caffeine. In contrast, carbamazepine, sulfamethoxazole, sulfapyridine and oxytetracycline appear more resistant to treatment whereas removal efficiency for novobiocin, trimethoprim and cotinine is either unclear or unknown. Despite the degree to which they are eliminated in WWTps, their presence in urban effluents discharging to surface waters is common. To estimate their potential risk to aquatic biota, we undertook acute/chronic toxicity testing with a suite of small-scale bioassays representing four taxonomic groups (bacteria, algae, invertebrates and fish) and complemented our results with those published in the literature. When all acute and chronic toxicity tests were combined, their toxicity responses spanned over five orders of magnitude. Barring caffeine and cotinine, all others are clearly recognized as toxic based on the EU-Directive 93/67/EEC classification, but their concentrations measured in WTTPs are too low to cause acute or chronic effects on an individual basis. Sulfamethoxazole, naproxen and ibuprofen, however, were found to produce toxic effects at concentrations that are $\leq$ one order of magnitude to those found in municipal effluents. In general, bioassay responses displayed a wide range of sensitivity that justifies continuing use of representative species within test batteries to properly appraise the toxic potential of pharmaceuticals. We also showed that exposure of Hydra attenuata to caffeine for $48 \mathrm{~h}$ induced xanthine oxidoreductase activity which may in turn lead to production of oxidative stress at concentrations close to those present in urban effluents. Future studies on the ecotoxicity of pharmaceuticals should therefore also seek to include appropriate biomarkers to assess possible adverse long-term effects to biota resulting from the biotransformation of drugs.
\end{abstract}

Keywords : pharmaceuticals, acute and chronic toxicity, municipal effluents, bioassays, biomarkers, Saint-Lawrence River.

\section{INTRODUCTION}

Historically known for introducing biological agents (bacteria, viruses, parasites, etc.), as well as several types of «classical» contaminants (i.e., metals and organics) to surface waters, municipal effluents are also now regarded as important sources of so-called «emerging chemicals» including household products, surfactant and pharmaceuticals (drugs and hormones).
The latter, either through original molecules or metabolites, are then released into the aquatic environment where their biological effects can potentially be exerted on biota. Clearly a source of concern with respect to environmental protection, this issue has been remarkably evoked in several recent reviews relating to drugged waters (Cunningham et al., 2006; Garric \& Ferrari, 2005; Halling-Sorensen et al., 1998; Kümmerer, 2001; Jones et al., 2001). 
Indeed, the presence of pharmaceuticals and personal care products is now documented for sewage, wastewater treatment plants and other (non)point sources of pollution (Stumpf et al, 1999; Jones et al., 2001; Kümmerer, 2001; Pedersen et al., 2005), as well as for surface (Dietrich et al., 2002; Metcalfe et al., 2003a; Gross et al., 2004; Kolpin et al., 2002) and even potable waters (Stackelberg et al., 2004). In the short term, pharmaceutical strains on aquatic systems will likely not diminish and may even be expected to increase owing to an aging human population and continuing efforts to develop new classes of therapeutic chemicals (Servos et al., 2002). In the long term, improved technology and efficiency of sewage wastewater treatment plants should eventually prevail to curb the entrance of undesirable drug products in receiving waters, as suggested by marked research presently on-going in this area (Heberer \& Feldmann, 2004; Perez et al., 2005; Roberts \& Thomas, 2006; Batt et al., 2006a,b; Jasim et al., 2006). For now, ecotoxicological studies, still relatively sparse, must continue to document hazard and risk potential for chemicals released to aquatic destinations by reporting their acute and chronic toxicities, both individually and in combination (Crane et al., 2006). Drugs displaying persistent characteristics will also merit additional investigations to assess their bioaccumulation and biomagnification profiles via trophic chain experimentation (Gagné et al., 2006c).

In the work presented herein, we report on several aspects linked to the ecotoxicity of 12 recently-quantified pharmaceuticals discharging to the freshwater portion of the St-Lawrence River in the Greater Montreal area via a major municipal wastewater treatment plant (WWTP) effluent. This WWTP, offering primary treatment with phosphorus removal, handles close to three million $\mathrm{m}^{3} / \mathrm{d}$ of wastewater (sewage combined with $10-20 \%$ industrial loading input), is known to release (in)organic contaminants (Pham \& Proulx, 1997; Quémerais et al., 1998) and has been shown to be toxic (Environment Canada, 1998), genotoxic (White, 1996), immunotoxic (Blaise et al., 2002), as well as being a source of estrogenic substances causing feminization effects in freswater bivalves (Gagné et al., 2001; Blaise et al., 2003).

Our study first investigated the effects of these 12 pharmaceuticals with a suite of small-scale toxicity tests (microbiotests) representing different levels of biological organization. In so doing, we also considered all other acute and chronic toxicity test data reported for these substances in the literature to determine their relative hazard potential in relation to risk for aquatic species. Beyond bioassays, we also discuss the more subtle adverse effects that can be associated with uptake and metabolization of pharmaceuticals. In this area, aspects of the biotransformation of caffeine by the cnidarian Hydra attenuata were explored.

\section{MATERIALS AND METHODS}

\section{Sampling of municipal effluent and pharmaceutical analysis}

Three $60 \mathrm{~L}$ grab samples were collected from the final wastewater treatment plant effluent (Montreal, Canada) in June 2003 and placed in Rubbermaid plastic containers (High density poly-ethylene; Rubbermaid commercial products, Mississauga, Ontario, Canada) lined with polyethylene bags. The samples were maintained at $4^{\circ} \mathrm{C}$ during transport to our laboratories with commercial ice-packs placed between the container and bag lining.

The effluent was analyzed for a total of 42 products: six neutral pharmaceuticals (trimethoprim, cotinine, pentoxifylline, carbamazepine, cyclophosphamide, and caffeine); nine acidic pharmaceuticals (bezafibrate, gemfibrozil, naproxen, clofibrate, diclofenac, fenoprofen, ibuprofen, indomethacin and ketoprofen); 18 sulfonamide antibiotics (sulfacetamide, sulfaguanidine, sulfapyridine, sulfadiazine, sulfamethoxazole, sulfathiazole, sulfamerazine, sulfasoxazole, sulfamoxole, sulfamethizole, sulfabenzamide, sulfamethazine, sulfamethoxypyridazine, sulfameter, sulfachloropyridazine, sulfadimethoxine, sulfaphenazole and sulfaquinoxaline); four quinolone antibiotics (norfloxacin, pipemidic acid, oxolinic acid, ofloxacin); three tetracyclines (oxytetracycline, chlorotetracycline and tetracycline) and two macrolide antibiotics (roxithromycin, novobiocin). Analytical techniques made use of high performance liquid chromatography-mass spectrometry (LC-MS/MS). They were performed by Enviro-Test Laboratories/Xenos Division, located in Ottawa (Ontario, Canada).

\section{Toxicity tests}

Six well-standardized bioassays representing four trophic levels were employed to assess the toxic potential of pharmaceutical products detected in the municipal wastewater treatment plant effluent. Their features are reported in Table 1. Listed references are links to the respective methods and statistical analyses that were followed to undertake testing.

\section{Biomarker measurement and statistical analysis \\ Hydra attenuata was cultivated under previously} reported conditions (Blaise and Kusui, 1997) and exposed to concentrations of caffeine for $48 \mathrm{~h}$ at $20^{\circ} \mathrm{C}$ at a density of 50 animals per treatment. At the end of 
the exposure period, 25 Hydra were collected with a Pasteur pipette, centriguged at $100 \mathrm{~g}$ for $5 \mathrm{~min}$ and the Hydra pellet homogenized with a Teflon pestle tissue grinder in $1 \mathrm{~mL}$ of $50 \mathrm{mM} \mathrm{NaCl}$ containing $10 \mathrm{mM}$ Hepes$\mathrm{NaoH}$, pH 7.4 containing $0.1 \mathrm{mM}$ EDTA. The homogenate was then centrifuged at $15000 \mathrm{~g}$ for $20 \mathrm{~min}$ at $4^{\circ} \mathrm{C}$ and the supernatant $\left(S_{15}\right.$ fraction) collected for analysis. The oxidative metabolism of xanthines was measured by following xanthine oxidoreductase (XOR) activity in the $S_{15}$ fraction by the method of Zhu et al. (1994). The mixture contained $50 \mu \mathrm{L}$ of $S_{15}$ fraction, 1 $\mathrm{mM}$ hypoxanthine, $0.1 \mathrm{mM}$ aminotriazole (a catalase inhibitor), $2 \mu \mathrm{M}$ dichlorofluorescein and $0.1 \mu \mathrm{g} / \mathrm{mL}$ horseradish peroxidase in $25 \mathrm{mM} \mathrm{KH}_{2} \mathrm{PO}_{4}, \mathrm{pH} 7.4$, containing $10 \mu \mathrm{M}$ molybdate. The reaction proceeded at $30^{\circ} \mathrm{C}$ for $0,15,30$ and 60 min and resulting production of fluorescein was measured under $485 \mathrm{~nm}$ excitation and $520 \mathrm{~nm}$ emission. Standard solutions of fluorescein were used for calibration. Enzyme activtiy was normalised against total protein in the $S_{15}$ fraction using serum bovine albumin as standard (Bradford, 1976). Enzyme activity was expressed as nmole of fluorescein formed $/ \mathrm{min} / \mathrm{mg}$ proteins. Differences between caffeine concentrations exposed to Hydra over controls were shown by ANOVA followed by the least square difference (Fisher) using Statistica software (Version 7).

\section{RESULTS AND DISCUSSION}

\section{Effluent concentrations of pharmaceuticals}

Of 42 pharmaceutical products analyzed for, 12 were found to be above instrumental detection limits in the municipal effluent. Their characteristics and

Table 1 - Characteristics of the small-scale bioassays used by the authors to determine the toxic potential of the 12 pharmaceuticals.

\begin{tabular}{|c|c|c|c|c|}
\hline Trophic level & Toxicity test & $\begin{array}{l}\text { Assessment } \\
\text { endpoint }\end{array}$ & $\begin{array}{l}\text { Measurement } \\
\text { endpoint }\end{array}$ & Reference \\
\hline Decomposer & $\begin{array}{l}\text { Bacterial test } \\
\text { Vibrio fischeri } \\
\text { (Microtox® } \\
\text { toxicity test) }\end{array}$ & $\begin{array}{l}\text { Acute sublethal } \\
\text { light inhibition } \\
\text { (after a 15-min } \\
\text { exposure) }\end{array}$ & $15 \mathrm{~min}-\mathrm{IC} 50$ & $\begin{array}{c}\text { Environment Canada, } \\
1992\end{array}$ \\
\hline Decomposer & $\begin{array}{l}\text { Bacterial test } \\
\text { Escherichia coli } \\
\text { (SOS Chromotest } \\
\text { genotoxicity } \\
\text { assay) }\end{array}$ & $\begin{array}{l}\text { SOS gene DNA } \\
\text { repair induction } \\
\text { with and without } \\
\text { metabolic } \\
\text { activation (after a } \\
\text { 2-h exposure) }\end{array}$ & 2 h-TEC ${ }^{a}$ & Legault et al., 1996 \\
\hline Primary producer & $\begin{array}{l}\text { Algal test } \\
\text { (Pseudokirchneri } \\
\text { ella subcapitata } \\
\text { microplate assay) }\end{array}$ & $\begin{array}{l}\text { Chronic sublethal } \\
\text { growth inhibition } \\
\text { (after a 72-h } \\
\text { exposure) }\end{array}$ & $72 h-I C 50$ & $\begin{array}{l}\text { Blaise and Vasseur, } \\
2005\end{array}$ \\
\hline $\begin{array}{l}\text { Primary } \\
\text { consumer }\end{array}$ & $\begin{array}{l}\text { Micro-crustacean } \\
\text { test } \\
\text { (ThamnoToxkit } \\
\text { assay) }\end{array}$ & $\begin{array}{l}\text { Acute lethality } \\
\text { (after a 24-h } \\
\text { exposure) }\end{array}$ & 24h-LC50 & $\begin{array}{c}\text { Microbiotests Inc., } \\
\text { http://www.microbiote } \\
\text { sts.be/ }\end{array}$ \\
\hline $\begin{array}{l}\text { Secondary } \\
\text { consumer }\end{array}$ & $\begin{array}{l}\text { Cnidarian test } \\
\text { (Hydra attenuata } \\
\text { assay) }\end{array}$ & $\begin{array}{l}\text { Acute sublethality } \\
\text { indicated by } \\
\text { morphology } \\
\text { changes (after a } \\
96-h \text { exposure) }\end{array}$ & 96h-EC50 & Blaise and Kusui, 1997 \\
\hline $\begin{array}{l}\text { Secondary } \\
\text { consumer }\end{array}$ & $\begin{array}{l}\text { Fish cell test } \\
\text { (rainbow trout } \\
\text { primary } \\
\text { hepatocyte test) }\end{array}$ & $\begin{array}{l}\text { Acute cytotoxicity } \\
\text { (after a 48-h } \\
\text { exposure) }\end{array}$ & $48 \mathrm{~h}-\mathrm{TEC}^{\mathrm{b}}$ & Gagné, 2005 \\
\hline
\end{tabular}

a) TEC (threshold effect concentration) for SOS gene induction as manifested by significant production of $\beta$-galactosidase $=(\text { NOEC } \times \text { LOEC })^{1 / 2}$, where NOEC $=$ no observed effect concentration and LOEC = lowest observed effect concentration. b) TEC (threshold effect concentration) for cytotoxicity as manifested by a significant reduction in cell viability $=(\mathrm{NOEC} x$ LOEC $)^{1 / 2}$, where NOEC = no observed effect concentration and LOEC = lowest observed effect concentration. 
concentrations are reported in Table 2. In general, measured concentrations are in agreement with those quantified in urban effluents elsewhere. Ibuprofen, a commonly prescribed non sterodial cyclooxygenase (COX) inhibitor preventing prostaglandin synthesis and hence blocking pain and inflammation (Vane \& Botting, 1998), has been reported in other Canadian and European municipal wastewaters at concentrations ranging from $1300-85000 \mathrm{ng} / \mathrm{L}$ (Andreozzi et al., 2003; Metcalfe et al., 2003b; la Farré et al., 2001; Stumpf et al., 1999; Ternes, 1998; Roberts \& Thomas, 2006). Effluents from hospitals can be important sources of ibuprofen and other drugs to WWTPs via municipal sewage collection systems (Gomez et al., 2006). Despite marked biodegradation (75-99\%) following its passage in wastewater treatment plants (Tixier et al., 2003; Buser et al., 1999; Paxéus, 2004; Roberts \& Thomas, 2006), ibuprofen is nevertheless very present in North American and European surface waters (41$9500 \mathrm{ng} / \mathrm{L}$ ), suggesting significant use by human consumers (Heberer et al., 2001; Ternes, 1998; Buser et al., 1999; Stumpf et al., 1999; la Farré et al., 2001; Kolpin et al., 2002). Its elimination after urban wastewater treatment has also been estimated to be $77.8 \%$ in South Korean wastewater sewage treatment plants (Han et al., 2006) and between 12-100\% for a series of plants located in cities of several countries where treatment, process and season of assessment differed (Fent et al., 2006).

Similarly, naproxen, also a COX inhibitor, has been found in urban effluents of large cities at concentrations between 168 - 33900 ng/L (Andreozzi et al., 2003; Metcalfe et al., 2003b; la Farré et al., 2001; Ternes, 1998; Tixier et al., 2003; Jones et al., 2002). Elimination of naproxen after urban wastewater treatment is estimated to be $66 \%$ (Ternes, 1998) owing to biodegradation and photo-transformation processes (Tixier et al., 2003). Again, its removal rate following passage through sewage plants based on varying treatment options has been shown to vary between 15$100 \%$ (Fent et al., 2006). Overall, less consumption of naproxen compared to ibuprofen (e.g., 35 tons versus 162 tons annually in the UK, respectively : Jones et al., 2002) might somewhat explain the lower concentrations observed in municipal effluent sources.

Acting as lipid regulators, gemfibrozil and bezafibate control blood levels of cholesterol and triglycerides to minimize cardiovascular risk. Both were detected in similar concentrations in the urban effluent under study (59 and 72 ng/L, respectively). Gemfibrozil

Table 2 - Classes and concentrations of the 12 pharmaceutical products detected in a major municipal wastewater treatment plant effluent located in the Montreal area.

\begin{tabular}{|c|c|c|c|}
\hline Class & Substance & Biological effect & $\begin{array}{l}\text { Detected } \\
\text { concentration } \\
(\mathrm{ng} / \mathrm{L})^{\mathrm{a}}\end{array}$ \\
\hline \multirow[t]{2}{*}{$\begin{array}{l}\text { Anti-inflammatory } \\
\text { agent }\end{array}$} & Ibuprofen & COX inhibitor & 1191 \\
\hline & Naproxen & COX inhibitor & 217 \\
\hline \multirow[t]{2}{*}{ Lipid regulator } & Gemfibrozil & PPAR $^{b}$ agonist & 59 \\
\hline & Bezafibrate & PPAR $^{b}$ agonist & 72 \\
\hline Anti-convulsant & Carbamazepine & $\begin{array}{l}\text { Narcosis (membrane } \\
\text { depolarisation) }\end{array}$ & 33 \\
\hline \multirow[t]{5}{*}{ Antibiotics } & Sulfamethoxazole & $\begin{array}{l}\text { Tetrahydrofolate synthesis } \\
\text { inhibitor }\end{array}$ & 99 \\
\hline & Sulfapyridine & $\begin{array}{l}\text { Competitive inhibitor of } \\
\text { bacterial PABA }\end{array}$ & 46 \\
\hline & Oxytetracycline & $\begin{array}{l}\text { Bacterial protein synthesis } \\
\text { inhibitor }\end{array}$ & 440 \\
\hline & Novobiocin & DNA synthesis inhibitor & 330 \\
\hline & Trimethoprim & $\begin{array}{l}\text { Prevents reduction of } \\
\text { dihydrofolate }\end{array}$ & 63 \\
\hline Stimulant & Caffeine & $\begin{array}{l}\mathrm{CNS}^{\mathrm{d}} \text {, cardiac and } \\
\text { respiratory stimulant }\end{array}$ & 22187 \\
\hline Nicotine metabolite & Cotinine & Nicotine metabolite & 236 \\
\hline
\end{tabular}

a) These recently-published data (Gagné et al., 2006a, Table 1, page 331) are reproduced by permission of Elsevier.

b) Peroxisome proliferator-activated receptor.

c) Para-aminobenzoic acid.

d) Central nervous system 
concentrations found in comparable wastewaters of other countries have been reported to be somewhat higher (1200 - $4760 \mathrm{ng} / \mathrm{L})$ than that measured in our effluent (Metcalfe et al., 2003b; Tixier et al., 2003; Andreozzi et al., 2003; Ternes, 1998). In their study of 18 Canadianbased wastewater treatment plants, Metcalfe et al. (2003b) failed to detect gemfibrozil in the same Montreal effluent $(<20 \mathrm{ng} / \mathrm{L}$ detection limit). Elimination of this drug by wastewater treatment has been purported to be in the order of $46 \%$ and $69 \%$ according to studies conducted in Brazil (Stumpf et al., 1999) and Germany (Ternes, 1998), respectively. Citing varied sources, Fent et al. (2006) report gemfibrozil removal rates to lie between $10-75 \%$. Variations in treatment plant performance may, among other factors, explain this discrepancy with respect to \% elimination. The bezafibrate concentration reported by Metcalfe et al. (2003b) in the same effluent was $600 \mathrm{ng} / \mathrm{L}$, a value eightfold higher than the one we report herein $(72 \mathrm{ng} / \mathrm{L})$. Other concentrations measured elsewhere in similar effluents range from 259 - $4600 \mathrm{ng} / \mathrm{L}$ (Metcalfe et al., 2003b; Ternes, 1998; Stumpf et al., 1999). Again, treatment plant efficiency to eliminate this product appears to vary between 50\% (Stumpf et al., 1999) and 83\% (Ternes, 1998). A more recent investigation indicates that bezafibrate removal, all factors confounded, can vary between 10-100\% (Fent et al., 2006).

Carbamazepine possesses anti-convulsant properties proven useful for treating epilepsy. It is also used to ease chronic pain (Hansen, 1999) and in psycho-pharmaco therapy to treat bipolar disorder (Bowden, 1997). Unlike most other medications, carbamazepine appears resistant to wastewater treatment and its elimination, $3 \%$ (Jones et al., 2002), $7 \%$ (Ternes, 1998), and < 10\% (Paxéus, 2004) is rather negligible. More recent studies, however, suggest that removal of this pharmaceutical by sewage treatment plants is improving : 0-53\% (Fent et al., 2006); 91.3\% (Han et al., 2006). Several of its chemical properties including lipophilicity $\left(\mathrm{K}_{\mathrm{ow}}=2.93\right.$, Kasim et al., 2004), half-life of 100 days (Ternes, 1998; Andreozzi et al., 2003) and biocencentration factor (15.36, Jones et al., 2002) suggest it could be persistent once it enters an aquatic environment and may have a potential for food chain bio-magnification that will merit further attention (Gagné et al., 2006c). In their study on the same Montreal effluent, Metcalfe et al. (2003b) reported a concentration for carbamazepine of $400 \mathrm{ng} / \mathrm{L}$, a value 12 -fold higher than ours (33 ng/L). Values determined elsewhere in similar effluents range from $126-6900$ $\mathrm{ng} / \mathrm{L}$ (Metcalfe et al., 2003b; Tixier et al., 2003; Andreozzi et al., 2003; Ternes, 1998; Zwiener \& Frimmel, 2004).

Five antibiotics were detected in the municipal effluent investigated (Table 2). Designed to inhibit microbial growth or to be biocidal, antibiotics are widely used for human and veterinary purposes. Examples of reported consumption indicate 10000 and 14000 tons per annum in Europe (Kummerer, 2001) and the United States of America (Sanderson et al., 2004). With regards to sulfonamide antibiotics, sulfamethoxazole concentrations determined in similar effluents in European cities ranged from $20-2000 \mathrm{ng} / \mathrm{L}$ (Hirsch et al. 1999; Andreozzi et al. 2003), a spread clearly influenced by factors such as population, consumption as well as wastewater treatment process and efficiency. For final WWTP effluents in Canada, median and maximum values of 243 and $871 \mathrm{ng} / \mathrm{L}$ have been reported, respectively (Miao et al., 2004). A more recent investigation conducted at three WWTPs with varying treatment technologies and design in the United States detected sulfamethoxazole concentrations ranging from 370 to $6000 \mathrm{ng} / \mathrm{L}$ (Batt et al., 2006a). A 1985 study suggests that sulfamethoxazole is poorly degradable (Richardson \& Brown, 1985), while a more recent one indicates the contrary based on its half-life of 2.4 days (Andreozzi et al., 2003). Other studies corroborate its poor degradability by sewage treatments (HallingSorensen, 1998) and its persistence in the environment (Jjemba, 2006). Our reported value of $46 \mathrm{ng} / \mathrm{L}$ in sewage effluent for sulfapyridine, a second sulfonamide antibiotic, could only be spatially compared with two other published studies. In WWTP effluents located in five Canadian cities, its concentrations averaged $81 \mathrm{ng} / \mathrm{L}$ with a maximum value of $228 \mathrm{ng} / \mathrm{L}$ (Miao et al., 2004). Concentrations ranging from $40-350 \mathrm{ng} / \mathrm{L}$ were also reported for two municipal WWTPs in Switzerland (Göbel et al., 2005). This same study also showed that elimination of sulfapyridine following activated sludge treatment is incomplete likely owing to the low sorption capacity of sulfonamides with solid particles (i.e., sorption constants for activated sludge below $500 \mathrm{~L} / \mathrm{kg}$ ).

Three other antibiotics were quantified in the Montreal urban effluent for which little or no other spatial data exist in terms of their presence in similar waste or surface waters. Oxytetracycline, a tetracycline antibiotic, widely used in the UK at 30 tons per annum (Jones et al., 2002; Schowanek \& Webb, 2000), is known to be resistant to biodegradation (Richardson and Brown, 1985; Jones et al., 2002), but likely not bioaccumulative owing to its low bioconcentration factor value of three (Jones et al., 2002). Its half-life under anoxic conditions is reported to be one of nine days (Halling-Sorensen et al., 1998). While there is a paucity of data on the presence of oxytetracycline in other sewage effluents, maximum concentrations of 340 and $1630 \mathrm{ng} / \mathrm{L}$ have been reported for oxytetracycline in surface waters of the U.S.A. (Kolpin et al., 2002) and the U.K. (Schowanek \& Webb, 2000), respectively. Total removal of this antibiotic by WWTP processes 
(biodegradation, sorption to sludge, etc.) has been estimated to be in the order of only $2 \%$ (Jones et al., 2002).

No spatial data were found for similar effluents with which to compare our reported concentration of $330 \mathrm{ng} / \mathrm{L}$ for the macrolide antibiotic, novobiocin (Table 2). Data on its persistence and degradability potential also appear absent in the literature. For the bacteriostatic antibiotic trimethoprim, quantified at $63 \mathrm{ng} / \mathrm{L}$ in our municipal wastewater (Table 2), an average concentration of $154 \mathrm{ng} / \mathrm{L}$ for other sewage treatment plant effluents has been reported (Hernando et al., 2006), as have maximum values of $660 \mathrm{ng} / \mathrm{L}$ in German WWTP effluents (Zwiener \& Frimmel, 2004) and of $1288 \mathrm{ng} / \mathrm{L}$ in U.K. WWTP effluents (Ashton et al., 2004). In another investigation, three grab samples taken at different times of the year in one WWTP effluent in North America revealed trimethoprim concentrations of 94,313 and 344 $\mathrm{ng} / \mathrm{L}$ (Hua et al., 2006). This study also demonstrated the marked influence that seasonality can have in modulating WWTP effluent pharmaceutical concentrations for trimethoprim and other substances owing to changes in water conditions. The fate of trimethoprim in WWTPs is unclear, but its environmental half-life has been reported to lie between 20-100 days (Zuccato et al., 2001). A recent study reports a WWTP removal efficiency of only three percent for trimethoprim based on a comparison of median concentration values measured in raw and final effluents (Roberts and Thomas, 2006). In contrast, nitrifying bacteria activated sludge was shown to enhance removal of trimethoprim in WWTP effluent up to $50 \%$ over conventional activated sludge at only $1 \%$ owing to higher solid retention time in the former sludge (Batt et al., 2006b).

As an alkaloid present in more than 60 plant species, e.g. tea, coffee, cacao (IARC, 1991), caffeine is likely the most consumed drug worldwide (Ogunseitan, 1996). Clearly beneficial as a cardiac, cerebral and respiratory stimulant, among other pharmaceutical properties, its presence appears ubiquitous in wastewaters, surface waters and groundwaters (Buerge et al., 2003). Indeed, it has been suggested as a suitable marker of anthropogenic inputs of domestic origin to aquatic ecosystems (Buerge et al., 2003; Siegener \& Chen, 2002). Wastewater treatment plant effluents in Switzerland are reported to discharge between 30-9500 ng/L of caffeine (Buerge et al., 2003) while concentrations emitted by similar effluents to Boston harbor lie between $370-6700 \mathrm{ng} / \mathrm{L}$ (Siegener \& Chen, 2002). The higher concentration found in the Montreal municipal effluent investigated (22187 $\mathrm{ng} / \mathrm{L}$, Table 2) may be linked to its limited treatment (primary treatment with phosphorus removal only), as caffeine appears to be more efficiently removed in domestic wastewaters receiving activated sludge treatment (Buerge et al., 2003) where it is readily biodegradable (Halling-Sorensen et al., 1998).

The nicotine metabolite cotinine was the 12th pharmaceutical product detected in the Montreal-based wastewater treatment plant (WWTP) effluent investigated at a concentration of $236 \mathrm{ng} / \mathrm{L}$ (Table 2). Cotinine is generally present in WWTP effluents and surface waters contaminated by drugs, and levels of 22 (mean) and 58 $\mathrm{ng} / \mathrm{L}$ (maximum) have been previously reported for Canadian WWTP effluents (Metcalfe et al. 2004). We have not found data relating to its persistence/ degradability potential nor removal efficiency by WWTPs in the scientific literature.

\section{Biological effects of pharmaceuticals}

Toxicity data determined in our study on the 12 pharmaceuticals integrated with those reported in the literature reflect a wide spectrum of sensitivity that is biological level-, test- and endpoint-specific (Table 3). Overall, toxicity testing performed elsewhere with acute/ chronic bioassays similar to ours essentially display commensurate levels of responses for these chemicals. Table 3 highlights their available acute and chronic effects that have been thus far generated worldwide at various levels of aquatic biological organization. Included are toxicity results for bacteria (acute), blue-green algae (chronic), algae (chronic), macrophytes (chronic), invertebrates (acute and chronic), as well as fish/fish cells (acute) and bivalve cells (acute). Notable is the recurrent use of several well-documented and popular bioassays (e.g., V. fischeriluminescence inhibition test, $P$. subcapitata growth inhibtion test, $D$. magna immobilization and reproduction tests) that have proven valuable to screen chemical toxicity in past decades, as well as more recent bioassays that show promise for such purposes (e.g., macrophyte growth inhibition tests, Hydra morphology and behavioral tests, fish and bivalve cell assays). With all acute and chronic tests confounded for these 12 pharmaceuticals, toxicity responses span over five orders of magnitude (least sensitive response : $24 \mathrm{~h}$ LC50 of the invertebrate $T$. platyurus $=4661 \mathrm{mg} / \mathrm{L}$ for caffeine; most sensitive response : $96 \mathrm{~h}$ IC50 of the cyanobacterium $S$. leopolensis $=0.027 \mathrm{mg} / \mathrm{L}$ for sulfamethoxazole). A similar spectrum of responses has been reported in a recent study of ecotoxicity data compiled for over 100 pharmaceuticals where acute toxicity effects spanned over six orders of magnitude (Webb, 2004).

Classifying bioassay data in Table 3 according to EU-Directive 93/67/EEC (CEC, 1996) offers some estimate of hazard potential for the pharmaceuticals studied (Table 4). While the spread of toxicity responses places these 12 compounds in all cut-off classes, some notable trends appear in their ecotoxic potency. Caffeine and cotinine are clearly less hazardous than all others. 
Braz. J. Aquat. Sci. Technol., 2006, 10(2):29-51.

Table 3 - Acute $(A)$ and chronic $(C)$ bioassay responses to the 12 pharmaceutical products investigated. Toxicity data are from our study and from those reported in the literature.

\begin{tabular}{|c|c|c|c|c|c|}
\hline Substance & $\begin{array}{l}\text { Reported } \\
\text { value } \\
\text { (mg/L) }\end{array}$ & $\begin{array}{l}\text { Measurement } \\
\text { endpoint }\end{array}$ & $\begin{array}{l}\text { Assessment } \\
\text { endpoint }\end{array}$ & Species & Reference \\
\hline \multicolumn{6}{|l|}{ Ibuprofen } \\
\hline \multirow[t]{3}{*}{ Bacteria } & 12.3 & $\begin{array}{l}5-\min \mathrm{IC} 50 \\
\text { (A) }\end{array}$ & $\begin{array}{l}\text { Luminescence } \\
\text { inhibition }\end{array}$ & Vibrio fischeri & Knoll, 1995 \\
\hline & 19.1 & $\begin{array}{l}15-\min I C 50 \\
\text { (A) }\end{array}$ & $\begin{array}{l}\text { Luminescence } \\
\text { inhibition }\end{array}$ & Vibrio fischeri & $\begin{array}{l}\text { la Farré et } \\
\text { al., } 2001\end{array}$ \\
\hline & 57.9 & $\begin{array}{l}\text { 15-min IC50 } \\
\text { (A) }\end{array}$ & $\begin{array}{l}\text { Luminescence } \\
\text { inhibition }\end{array}$ & Vibrio fischeri & This study \\
\hline \multirow[t]{4}{*}{ Algae } & 1 & $5 d-I C 25(C)$ & $\begin{array}{l}\text { Growth } \\
\text { inhibition }\end{array}$ & $\begin{array}{l}\text { Synechosystis sp. } \\
\text { (cyanobacterium) }\end{array}$ & $\begin{array}{l}\text { Pomati et } \\
\text { al., } 2004\end{array}$ \\
\hline & 90.5 & $72 \mathrm{~h}-\mathrm{IC} 50(\mathrm{C})$ & $\begin{array}{l}\text { Growth } \\
\text { inhibition }\end{array}$ & $\begin{array}{l}\text { Pseudokirchneriella } \\
\text { subcapitata }\end{array}$ & This study \\
\hline & 315 & $72 \mathrm{~h}-\mathrm{IC50}(\mathrm{C})$ & $\begin{array}{l}\text { Growth rate } \\
\text { inhibition }\end{array}$ & $\begin{array}{l}\text { Desmodesmus } \\
\text { subspicatus }\end{array}$ & $\begin{array}{l}\text { Cleuvers, } \\
2003\end{array}$ \\
\hline & 342 & $72 \mathrm{~h}-\mathrm{IC} 50(\mathrm{C})$ & $\begin{array}{l}\text { Growth rate } \\
\text { inhibition }\end{array}$ & $\begin{array}{l}\text { Desmodesmus } \\
\text { subspicatus }\end{array}$ & $\begin{array}{l}\text { Cleuvers, } \\
2004\end{array}$ \\
\hline \multirow[t]{3}{*}{ Macrophytes } & $>1$ & 7d-NOEC (C) & $\begin{array}{l}\text { Growth } \\
\text { inhibition }\end{array}$ & Lemna gibba & $\begin{array}{l}\text { Brain et al. } \\
2004\end{array}$ \\
\hline & 4.0 & 7d-IC50 (C) & $\begin{array}{l}\text { Growth } \\
\text { inhibition }\end{array}$ & Lemna gibba & $\begin{array}{l}\text { Pomati et } \\
\text { al., } 2004\end{array}$ \\
\hline & 22 & $7 d-I C 50(C)$ & $\begin{array}{l}\text { Growth rate } \\
\text { inhibition }\end{array}$ & Lemna minor & $\begin{array}{l}\text { Cleuvers, } \\
2003\end{array}$ \\
\hline \multirow[t]{8}{*}{$\begin{array}{l}\text { Micro- } \\
\text { invertebrates }\end{array}$} & $>0.01$ & 7d-LOEC (C) & $\begin{array}{l}\text { (Sub)lethal } \\
\text { toxicity and } \\
\text { feeding } \\
\text { inhibition }\end{array}$ & Hydra vulgaris & $\begin{array}{l}\text { Pascoe et } \\
\text { al., } 2003\end{array}$ \\
\hline & $\begin{array}{l}9.1 \text { to } \\
11.5\end{array}$ & 48h-EC50 (A) & Immobilization & Daphnia magna & Knoll, 1995 \\
\hline & 17.5 & 96h-EC50 (A) & $\begin{array}{l}\text { Morphology } \\
\text { perturbation }\end{array}$ & Hydra attenuata & This study \\
\hline & 20 & $\begin{array}{l}\text { 21d-NOEC } \\
(\mathrm{C})\end{array}$ & $\begin{array}{l}\text { Reproduction } \\
\text { inhibition }\end{array}$ & Daphnia magna & $\begin{array}{l}\text { Han et al., } \\
2006\end{array}$ \\
\hline & 108 & 48h-EC50 (A) & Immobilization & Daphnia magna & $\begin{array}{l}\text { Cleuvers, } \\
2003\end{array}$ \\
\hline & 132.6 & 48h-EC50 (A) & Immobilization & Daphnia magna & $\begin{array}{l}\text { Han et al., } \\
2006\end{array}$ \\
\hline & $>200$ & $24 h-L C 50(A)$ & Mortality & $\begin{array}{l}\text { Thamnocephalus } \\
\text { platyurus }\end{array}$ & $\begin{array}{l}\text { Nalecz- } \\
\text { Jawecki } \\
\text { and } \\
\text { Persoone, } \\
2006\end{array}$ \\
\hline & 471 & $24 \mathrm{~h}-\mathrm{LC} 50(\mathrm{~A})$ & Mortality & $\begin{array}{l}\text { Thamnocephalus } \\
\text { platyurus }\end{array}$ & This study \\
\hline \multirow[t]{4}{*}{ Fish/fish cells } & 173 & 96h-LC50 (A) & Mortality & $\begin{array}{l}\text { Lepomis } \\
\text { machrochirus } \\
\text { (bluegill sunfish) }\end{array}$ & Knoll, 1995 \\
\hline & $>228$ & $48 \mathrm{~h}-\mathrm{TEC}^{\mathrm{a}}(\mathrm{A})$ & Cytotoxicity & $\begin{array}{l}\text { Oncorhynchus } \\
\text { mykiss (primary } \\
\text { hepatocytes) }\end{array}$ & This study \\
\hline & 248 & 24h-EC50 (A) & Cytotoxicity & $\begin{array}{l}\text { PLHC-1 } \\
\text { (Poeciliopsis lucida) } \\
\text { hepatoma cells }\end{array}$ & $\begin{array}{l}\text { Caminada } \\
\text { et al., } 2006\end{array}$ \\
\hline & 1194 & 24h-EC50 (A) & Cytotoxicity & $\begin{array}{l}\text { RTG-2 (rainbow } \\
\text { trout) gonad cells }\end{array}$ & $\begin{array}{l}\text { Caminada } \\
\text { et al., } 2006\end{array}$ \\
\hline
\end{tabular}


Blaise et al.: Ecotoxicity of pharmaceuticals of urban origin.

Table 3 - (continuation)

\begin{tabular}{|c|c|c|c|c|c|}
\hline Substance & $\begin{array}{l}\text { Reported } \\
\text { value } \\
\text { (mg/L) }\end{array}$ & $\begin{array}{l}\text { Measurement } \\
\text { endpoint }\end{array}$ & $\begin{array}{l}\text { Assessment } \\
\text { endpoint }\end{array}$ & Species & Reference \\
\hline \multicolumn{6}{|l|}{ Naproxen } \\
\hline \multirow[t]{2}{*}{ Bacteria } & $>12.5$ & $\begin{array}{l}\text { 15-min IC50 } \\
\text { (A) }\end{array}$ & $\begin{array}{l}\text { Luminescence } \\
\text { inhibition }\end{array}$ & Vibrio fischeri & This study \\
\hline & 35.6 & $\begin{array}{l}15-\min \mathrm{IC} 50 \\
\text { (A) }\end{array}$ & $\begin{array}{l}\text { Luminescence } \\
\text { inhibition }\end{array}$ & Vibrio fischeri & $\begin{array}{l}\text { la Farré et } \\
\text { al., } 2001\end{array}$ \\
\hline \multirow[t]{4}{*}{ Algae } & 19.3 & 72h-IC50 (C) & $\begin{array}{l}\text { Growth } \\
\text { inhibition }\end{array}$ & $\begin{array}{l}\text { Pseudokirchneriella } \\
\text { subcapitata }\end{array}$ & This study \\
\hline & 31.8 & 96h-IC50 (C) & $\begin{array}{l}\text { Growth } \\
\text { inhibition }\end{array}$ & $\begin{array}{l}\text { Pseudokirchneriella } \\
\text { subcapitata }\end{array}$ & $\begin{array}{l}\text { Isidori et al., } \\
2005\end{array}$ \\
\hline & $>320$ & 72h-IC50 (C) & $\begin{array}{l}\text { Growth rate } \\
\text { inhibition }\end{array}$ & $\begin{array}{l}\text { Desmodesmus } \\
\text { subspicatus }\end{array}$ & $\begin{array}{l}\text { Cleuvers, } \\
2003\end{array}$ \\
\hline & 626 & $72 \mathrm{~h}-\mathrm{IC} 50(\mathrm{C})$ & $\begin{array}{l}\text { Growth rate } \\
\text { inhibition }\end{array}$ & $\begin{array}{l}\text { Desmodesmus } \\
\text { subspicatus }\end{array}$ & $\begin{array}{l}\text { Cleuvers, } \\
2004\end{array}$ \\
\hline \multirow[t]{2}{*}{ Macrophytes } & 1 & 7d-LOEC (C) & $\begin{array}{l}\text { Growth } \\
\text { inhibition }\end{array}$ & Lemna gibba & $\begin{array}{l}\text { Brain et al. } \\
2004\end{array}$ \\
\hline & 24.2 & $7 d-I C 50(C)$ & $\begin{array}{l}\text { Growth rate } \\
\text { inhibition }\end{array}$ & Lemna minor & $\begin{array}{l}\text { Cleuvers, } \\
2003\end{array}$ \\
\hline \multirow[t]{12}{*}{$\begin{array}{l}\text { Micro- } \\
\text { invertebrates }\end{array}$} & 0.33 & 7d-EC50 (C) & $\begin{array}{l}\text { Reproduction } \\
\text { inhibition }\end{array}$ & Ceriodaphnia dubia & $\begin{array}{l}\text { Isidori et al., } \\
2005\end{array}$ \\
\hline & 0.56 & 48h-EC50 (C) & $\begin{array}{l}\text { Reproduction } \\
\text { inhibition }\end{array}$ & $\begin{array}{l}\text { Brachionus } \\
\text { calyciflorus }\end{array}$ & $\begin{array}{l}\text { Isidori et al., } \\
2005\end{array}$ \\
\hline & 2.6 & 96h-EC50 (A) & $\begin{array}{l}\text { Morphology } \\
\text { perturbation }\end{array}$ & Hydra attenuata & This study \\
\hline & $>25$ & 24h-LC50 (A) & Mortality & $\begin{array}{l}\text { Thamnocephalus } \\
\text { platyurus }\end{array}$ & This study \\
\hline & 62.5 & 24h-LC50 (A) & Mortality & $\begin{array}{l}\text { Brachionus } \\
\text { calyciflorus }\end{array}$ & $\begin{array}{l}\text { Isidori et al., } \\
2005\end{array}$ \\
\hline & 66.4 & 48h-LC50 (A) & Immobilization & Ceriodaphnia dubia & $\begin{array}{l}\text { Isidori et al., } \\
2005\end{array}$ \\
\hline & 84.1 & 24h-LC50 (A) & Mortality & $\begin{array}{l}\text { Thamnocephalus } \\
\text { platyurus }\end{array}$ & $\begin{array}{l}\text { Isidori et al., } \\
2005\end{array}$ \\
\hline & 140 & 24h-EC50 (A) & Immobilization & Daphnia magna & Webb, 2004 \\
\hline & 166 & 48h-EC50 (A) & Immobilization & Daphnia magna & $\begin{array}{l}\text { Cleuvers, } \\
2004\end{array}$ \\
\hline & 174 & 48h-EC50 (A) & Immobilization & Daphnia magna & $\begin{array}{l}\text { Cleuvers, } \\
2003\end{array}$ \\
\hline & $>200$ & 24h-LC50 (A) & Mortality & $\begin{array}{l}\text { Thamnocephalus } \\
\text { platyurus }\end{array}$ & $\begin{array}{l}\text { Nalecz- } \\
\text { Jawecki } \\
\text { and } \\
\text { Persoone, } \\
2006\end{array}$ \\
\hline & 383 & 96h-LC50 (A) & Mortality & Hyalella azteca & Webb, 2004 \\
\hline \multirow[t]{5}{*}{ Fish/fish cells } & $>230$ & $48 \mathrm{~h}-\operatorname{TEC}^{\mathrm{a}}(\mathrm{A})$ & Cytotoxicity & $\begin{array}{l}\text { Oncorhynchus } \\
\text { mykiss (primary } \\
\text { hepatocytes) }\end{array}$ & This study \\
\hline & 585 & 24h-EC50 (A) & Cytotoxicity & $\begin{array}{l}\text { PLHC-1 } \\
\text { (Poeciliopsis lucida) } \\
\text { hepatoma cells }\end{array}$ & $\begin{array}{l}\text { Caminada } \\
\text { et al., } 2006\end{array}$ \\
\hline & 560 & 96h-LC50 (A) & Mortality & $\begin{array}{l}\text { Lepomis } \\
\text { machrochirus }\end{array}$ & Webb, 2004 \\
\hline & 690 & 96h-LC50 (A) & Mortality & $\begin{array}{l}\text { Oncorhynchus } \\
\text { mykiss }\end{array}$ & Webb, 2004 \\
\hline & 1032 & 24h-EC50 (A) & Cytotoxicity & $\begin{array}{l}\text { RTG-2 (rainbow } \\
\text { trout) cells }\end{array}$ & $\begin{array}{l}\text { Caminada } \\
\text { et al., } 2006\end{array}$ \\
\hline
\end{tabular}


Braz. J. Aquat. Sci. Technol., 2006, 10(2):29-51.

Table 3 - (continuation).

\begin{tabular}{|c|c|c|c|c|c|}
\hline Substance & $\begin{array}{l}\text { Reported } \\
\text { value } \\
\text { (mg/L) }\end{array}$ & $\begin{array}{l}\text { Measurement } \\
\text { endpoint }\end{array}$ & $\begin{array}{l}\text { Assessment } \\
\text { endpoint }\end{array}$ & Species & Reference \\
\hline \multicolumn{6}{|l|}{ Gemfibrozil } \\
\hline \multirow[t]{3}{*}{ Bacteria } & $>15.6$ & $\begin{array}{l}\text { 15-min IC50 } \\
\text { (A) }\end{array}$ & $\begin{array}{l}\text { Luminescence } \\
\text { inhibition }\end{array}$ & Vibrio fischeri & This study \\
\hline & 31.5 & $\begin{array}{l}15-\min \mathrm{IC} 50 \\
\text { (A) }\end{array}$ & $\begin{array}{l}\text { Luminescence } \\
\text { inhibition }\end{array}$ & Vibrio fischeri & $\begin{array}{l}\text { la Farré et } \\
\text { al., } 2001\end{array}$ \\
\hline & 45.1 & $\begin{array}{l}15-\min I C 50 \\
\text { (A) }\end{array}$ & $\begin{array}{l}\text { Luminescence } \\
\text { inhibition }\end{array}$ & Vibrio fischeri & $\begin{array}{l}\text { Zurita et } \\
\text { al., } 2006\end{array}$ \\
\hline \multirow[t]{2}{*}{ Algae } & 7.3 & $72 \mathrm{~h}-\mathrm{IC50}(\mathrm{C})$ & $\begin{array}{l}\text { Growth } \\
\text { inhibition }\end{array}$ & $\begin{array}{l}\text { Pseudokirchneriella } \\
\text { subcapitata }\end{array}$ & This study \\
\hline & 150 & 72h-IC50 (C) & $\begin{array}{l}\text { Growth } \\
\text { inhibition }\end{array}$ & Chlorella vulgaris & $\begin{array}{l}\text { Zurita et } \\
\text { al., } 2006\end{array}$ \\
\hline \multirow[t]{4}{*}{$\begin{array}{l}\text { Micro- } \\
\text { invertebrates }\end{array}$} & 0.8 & 96h-EC50 (A) & $\begin{array}{l}\text { Morphology } \\
\text { perturbation }\end{array}$ & Hydra attenuata & This study \\
\hline & 10.4 & 48h-EC50 (A) & Immobilization & Daphnia magna & $\begin{array}{l}\text { Han et al., } \\
2006\end{array}$ \\
\hline & 30 & 72h-EC50 (A) & Immobilization & Daphnia magna & $\begin{array}{l}\text { Zurita et } \\
\text { al., } 2006\end{array}$ \\
\hline & $>31.3$ & 24h-LC50 (A) & Mortality & $\begin{array}{l}\text { Thamnocephalus } \\
\text { platyurus }\end{array}$ & This study \\
\hline \multirow[t]{3}{*}{ Fish/fish cells } & 219 & 24h-EC50 (A) & Cytotoxicity & $\begin{array}{l}\text { PLHC-1 } \\
\text { (Poeciliopsis lucida) } \\
\text { hepatoma cells }\end{array}$ & $\begin{array}{l}\text { Caminada } \\
\text { et al., } 2006\end{array}$ \\
\hline & $>250$ & $48 \mathrm{~h}-\operatorname{TEC}^{\mathrm{a}}(\mathrm{A})$ & Cytotoxicity & $\begin{array}{l}\text { Oncorhynchus } \\
\text { mykiss (primary } \\
\text { hepatocytes) }\end{array}$ & This study \\
\hline & 986 & 24h-EC50 (A) & Cytotoxicity & $\begin{array}{l}\text { RTG-2 (rainbow } \\
\text { trout) cells }\end{array}$ & $\begin{array}{l}\text { Caminada } \\
\text { et al., } 2006\end{array}$ \\
\hline \multicolumn{6}{|l|}{ Bezafibrate } \\
\hline Bacteria & $>15.6$ & $\begin{array}{l}\text { 15-min IC50 } \\
\text { A) }\end{array}$ & $\begin{array}{l}\text { Luminescence } \\
\text { inhibition }\end{array}$ & Vibrio fischeri & This study \\
\hline Algae & $>31.3$ & 72h-IC50 (C) & $\begin{array}{l}\text { Growth } \\
\text { inhibition }\end{array}$ & $\begin{array}{l}\text { Pseudokirchneriella } \\
\text { subcapitata }\end{array}$ & This study \\
\hline \multirow[t]{3}{*}{$\begin{array}{l}\text { Micro- } \\
\text { invertebrates }\end{array}$} & 5.3 & 96h-EC50 (A) & $\begin{array}{l}\text { Morphology } \\
\text { perturbation }\end{array}$ & Hydra attenuata & This study \\
\hline & 30.3 & 48h-EC50 (A) & Immobilization & Daphnia magna & $\begin{array}{l}\text { Han et al., } \\
2006\end{array}$ \\
\hline & $>62.5$ & 24h-LC50 (A) & Mortality & $\begin{array}{l}\text { Thamnocephalus } \\
\text { platyurus }\end{array}$ & This study \\
\hline \multirow[t]{3}{*}{ Fish/fish cells } & $>362$ & $48 \mathrm{~h}-\mathrm{TEC}^{\mathrm{a}}(\mathrm{A})$ & Cytotoxicity & $\begin{array}{l}\text { Oncorhynchus } \\
\text { mykiss (primary } \\
\text { hepatocytes) }\end{array}$ & This study \\
\hline & 445 & 24h-EC50 (A) & Cytotoxicity & $\begin{array}{l}\text { RTG-2 (rainbow } \\
\text { trout) cells }\end{array}$ & $\begin{array}{l}\text { Caminada } \\
\text { et al., } 2006\end{array}$ \\
\hline & 941 & 24h-EC50 (A) & Cytotoxicity & $\begin{array}{l}\text { PLHC-1 } \\
\text { (Poeciliopsis lucida) } \\
\text { hepatoma cells }\end{array}$ & $\begin{array}{l}\text { Caminada } \\
\text { et al., } 2006\end{array}$ \\
\hline \multicolumn{6}{|l|}{ Carbamazepine } \\
\hline \multirow[t]{3}{*}{ Bacteria } & 78.5 & $\begin{array}{l}\text { 15-min IC50 } \\
\text { A) }\end{array}$ & $\begin{array}{l}\text { Luminescence } \\
\text { inhibition }\end{array}$ & Vibrio fischeri & $\begin{array}{l}\text { Jos et al., } \\
2003\end{array}$ \\
\hline & $>125$ & $\begin{array}{l}\text { 15-min IC50 } \\
\text { A) }\end{array}$ & $\begin{array}{l}\text { Luminescence } \\
\text { inhibition }\end{array}$ & Vibrio fischeri & This study \\
\hline & $>810$ & $\begin{array}{l}\text { 30-min } \\
\text { IC50 (A) }\end{array}$ & $\begin{array}{l}\text { Luminescence } \\
\text { inhibition }\end{array}$ & Vibrio fischeri & $\begin{array}{l}\text { Ferrari et al., } \\
2004\end{array}$ \\
\hline
\end{tabular}


Blaise et al.: Ecotoxicity of pharmaceuticals of urban origin.

Table 3 - (continuation).

\begin{tabular}{|c|c|c|c|c|c|}
\hline Substance & \begin{tabular}{|l} 
Reported \\
value \\
(mg/L)
\end{tabular} & $\begin{array}{l}\text { Measurement } \\
\text { endpoint }\end{array}$ & $\begin{array}{l}\text { Assessment } \\
\text { endpoint }\end{array}$ & Species & Reference \\
\hline \multirow[t]{6}{*}{ Algae } & 31.6 & 96h-IC50 (C) & \begin{tabular}{|l} 
Growth \\
inhibition
\end{tabular} & $\begin{array}{l}\text { Cyclotella } \\
\text { meneghiniana } \\
\text { (diatom) }\end{array}$ & $\begin{array}{l}\text { Ferrari et } \\
\text { al., } 2004\end{array}$ \\
\hline & 33.6 & $96 h-I C 50(C)$ & $\begin{array}{l}\text { Growth } \\
\text { inhibition }\end{array}$ & $\begin{array}{l}\text { Synechococcus } \\
\text { leopolensis } \\
\text { (cyanobacterium) }\end{array}$ & $\begin{array}{l}\text { Ferrari et } \\
\text { al., } 2004\end{array}$ \\
\hline & 36.6 & $48 \mathrm{~h}-\mathrm{IC} 50(\mathrm{C})$ & \begin{tabular}{|l|l|} 
Growth \\
inhibition
\end{tabular} & Chlorella vulgaris & $\begin{array}{l}\text { Jos et al., } \\
2003\end{array}$ \\
\hline & 74 & 72h-IC50 (C) & $\begin{array}{l}\text { Growth rate } \\
\text { inhibition }\end{array}$ & $\begin{array}{l}\text { Desmodesmus } \\
\text { subspicatus }\end{array}$ & $\begin{array}{l}\text { Cleuvers, } \\
2003\end{array}$ \\
\hline & 79.4 & $72 h-I C 50(C)$ & $\begin{array}{l}\text { Growth } \\
\text { inhibition }\end{array}$ & $\begin{array}{l}\text { Pseudokirchneriella } \\
\text { subcapitata }\end{array}$ & This study \\
\hline & $>100$ & $96 h-I C 50(C)$ & \begin{tabular}{|l|} 
Growth \\
inhibition \\
\end{tabular} & $\begin{array}{l}\text { Pseudokirchneriella } \\
\text { subcapitata }\end{array}$ & $\begin{array}{l}\text { Ferrari et } \\
\text { al., } 2004\end{array}$ \\
\hline \multirow[t]{2}{*}{ Macrophytes } & $>1$ & 7d-EC10 (C) & \begin{tabular}{|l|} 
Growth \\
inhibition
\end{tabular} & Lemna gibba & $\begin{array}{l}\text { Brain et } \\
\text { al. } 2004\end{array}$ \\
\hline & 25.5 & $7 d-I C 50(C)$ & $\begin{array}{l}\text { Growth rate } \\
\text { inhibition }\end{array}$ & Lemna minor & $\begin{array}{l}\text { Cleuvers, } \\
2003 \\
\end{array}$ \\
\hline \multirow[t]{8}{*}{$\begin{array}{l}\text { Micro- } \\
\text { invertebrates }\end{array}$} & 12.1 & 96h-EC50 (A) & \begin{tabular}{|l|} 
Morphology \\
perturbation
\end{tabular} & Hydra attenuata & This study \\
\hline & $>13.8$ & 48h-EC50 (A) & Immobilization & Daphnia magna & $\begin{array}{l}\text { Ferrari et } \\
\text { al., } 2004\end{array}$ \\
\hline & 77.7 & 48h-EC50 (A) & Immobilization & $\begin{array}{l}\text { Ceriodaphnia } \\
\text { magna }\end{array}$ & $\begin{array}{l}\text { Ferrari et } \\
\text { al., } 2004\end{array}$ \\
\hline & 97.8 & 48h-EC50 (A) & Immobilization & Daphnia magna & $\begin{array}{l}\text { Jos et al., } \\
2003\end{array}$ \\
\hline & $>100$ & 48h-EC50 (A) & Immobilization & Daphnia magna & $\begin{array}{l}\text { Cleuvers, } \\
2003\end{array}$ \\
\hline & 111 & 48h-EC50 (A) & Immobilization & Daphnia magna & $\begin{array}{l}\text { Han et al., } \\
2006\end{array}$ \\
\hline & 140 & 24h-LC50 (A) & Mortality & $\begin{array}{l}\text { Thamnocephalus } \\
\text { platyurus }\end{array}$ & $\begin{array}{l}\text { Nalecz- } \\
\text { Jawecki } \\
\text { and } \\
\text { Persoone, } \\
2006\end{array}$ \\
\hline & $>250$ & 24h-LC50 (A) & Mortality & $\begin{array}{l}\text { Thamnocephalus } \\
\text { platyurus }\end{array}$ & This study \\
\hline \multirow[t]{3}{*}{ Fish/fish cells } & 21.3 & $48 \mathrm{~h}-\operatorname{TEC}^{a}(\mathrm{~A})$ & Cytotoxicity & $\begin{array}{l}\text { Oncorhynchus } \\
\text { mykiss (primary } \\
\text { hepatocytes) }\end{array}$ & This study \\
\hline & $>591$ & 24h-EC50 (A) & Cytotoxicity & $\begin{array}{l}\text { PLHC-1 } \\
\text { (Poeciliopsis } \\
\text { lucida) hepatoma } \\
\text { cells }\end{array}$ & $\begin{array}{l}\text { Caminada } \\
\text { et al., } \\
2006\end{array}$ \\
\hline & $>591$ & 24h-EC50 (A) & Cytotoxicity & $\begin{array}{l}\text { RTG-2 (rainbow } \\
\text { trout) cells }\end{array}$ & $\begin{array}{l}\text { Caminada } \\
\text { et al., } \\
2006\end{array}$ \\
\hline \multicolumn{6}{|l|}{ Sulfamethoxazole } \\
\hline \multirow[t]{2}{*}{ Bacteria } & 65.3 & $\begin{array}{l}15-\min \text { IC50 } \\
\text { (A) }\end{array}$ & $\begin{array}{l}\text { Luminescence } \\
\text { inhibition }\end{array}$ & Vibrio fischeri & This study \\
\hline & $>84$ & $\begin{array}{l}15-\min I C 50 \\
(\mathrm{~A})\end{array}$ & $\begin{array}{l}\text { Luminescence } \\
\text { inhibition }\end{array}$ & Vibrio fischeri & $\begin{array}{l}\text { Ferrari et } \\
\text { al., } 2004\end{array}$ \\
\hline
\end{tabular}


Braz. J. Aquat. Sci. Technol., 2006, 10(2):29-51.

Table 3 - (continuation).

\begin{tabular}{|c|c|c|c|c|c|}
\hline Substance & $\begin{array}{l}\text { Reported } \\
\text { value } \\
\text { (mg/L) }\end{array}$ & $\begin{array}{l}\text { Measurement } \\
\text { endpoint }\end{array}$ & $\begin{array}{l}\text { Assessment } \\
\text { endpoint }\end{array}$ & Species & Reference \\
\hline \multirow[t]{5}{*}{ Algae } & 0.027 & 96h-IC50 (C) & $\begin{array}{l}\text { Growth } \\
\text { inhibition }\end{array}$ & $\begin{array}{l}\text { Synechococcus } \\
\text { leopolensis } \\
\text { (cyanobacterium) }\end{array}$ & $\begin{array}{l}\text { Ferrari et } \\
\text { al., } 2004\end{array}$ \\
\hline & 0,146 & $96 h-I C 50(C)$ & \begin{tabular}{|l|l} 
Growth \\
inhibition
\end{tabular} & $\begin{array}{l}\text { Pseudokirchneriella } \\
\text { subcapitata }\end{array}$ & $\begin{array}{l}\text { Ferrari et } \\
\text { al., } 2004\end{array}$ \\
\hline & 1.53 & 72h-IC50 (C) & $\begin{array}{l}\text { Growth } \\
\text { inhibition }\end{array}$ & $\begin{array}{l}\text { Pseudokirchneriella } \\
\text { subcapitata }\end{array}$ & $\begin{array}{l}\text { Eguchi et } \\
\text { al., } 2004\end{array}$ \\
\hline & 2.4 & $96 h-I C 50(C)$ & $\begin{array}{l}\text { Growth } \\
\text { inhibition }\end{array}$ & $\begin{array}{l}\text { Cyclotella } \\
\text { meneghiniana } \\
\text { (diatom) }\end{array}$ & $\begin{array}{l}\text { Ferrari et } \\
\text { al., } 2004\end{array}$ \\
\hline & 13.4 & 72h-IC50 (C) & \begin{tabular}{|l|} 
Growth \\
inhibition
\end{tabular} & $\begin{array}{l}\text { Pseudokirchneriella } \\
\text { subcapitata }\end{array}$ & This study \\
\hline \multirow[t]{5}{*}{$\begin{array}{l}\text { Micro- } \\
\text { invertebrates }\end{array}$} & $>0.1$ & $6 \mathrm{~d}-\mathrm{EC} 50(\mathrm{~A})$ & Immobilization & Daphnia magna & $\begin{array}{l}\text { Flaherty } \\
\text { and } \\
\text { Dodson, } \\
2005\end{array}$ \\
\hline & 19.3 & 96h-EC50 (A) & $\begin{array}{l}\text { Morphology } \\
\text { perturbation }\end{array}$ & Hydra attenuata & This study \\
\hline & $>100$ & 48h-EC50 (A) & Immobilization & Daphnia magna & $\begin{array}{l}\text { Ferrari et } \\
\text { al., } 2004\end{array}$ \\
\hline & $>100$ & 48h-EC50 (A) & Immobilization & $\begin{array}{l}\text { Ceriodaphnia } \\
\text { magna }\end{array}$ & $\begin{array}{l}\text { Ferrari et } \\
\text { al., } 2004\end{array}$ \\
\hline & $>250$ & $24 \mathrm{~h}-\mathrm{LC} 50(\mathrm{~A})$ & Mortality & $\begin{array}{l}\text { Thamnocephalus } \\
\text { platyurus }\end{array}$ & This study \\
\hline Fish/fish cells & $>253$ & $48 \mathrm{~h}-\mathrm{TEC}^{\mathrm{a}}(\mathrm{A})$ & Cytotoxicity & $\begin{array}{l}\text { Oncorhynchus } \\
\text { mykiss (primary } \\
\text { hepatocytes) }\end{array}$ & This study \\
\hline Bivalve cells & 70.9 & $24 \mathrm{~h}-\operatorname{TEC}^{\mathrm{e}}(\mathrm{A})$ & $\begin{array}{l}\text { Phagocytosis } \\
\text { inhibition }\end{array}$ & $\begin{array}{l}\text { Hemocytes of } \\
\text { Elliptio complanata } \\
\text { (bivalve) }\end{array}$ & $\begin{array}{l}\text { Gagné et } \\
\text { al., 2006b }\end{array}$ \\
\hline \multicolumn{6}{|l|}{ Sulfapyridine } \\
\hline Bacteria & $>125$ & $\begin{array}{l}15-\min \text { IC50 } \\
\text { (A) }\end{array}$ & $\begin{array}{l}\text { Luminescence } \\
\text { inhibition }\end{array}$ & Vibrio fischeri & This study \\
\hline Algae & 10.2 & 72h-IC50 (C) & \begin{tabular}{|l|l|} 
Growth \\
inhibition
\end{tabular} & $\begin{array}{l}\text { Pseudokirchneriella } \\
\text { subcapitata }\end{array}$ & This study \\
\hline \multirow[t]{2}{*}{$\begin{array}{l}\text { Micro- } \\
\text { invertebrates }\end{array}$} & 144.4 & $24 \mathrm{~h}-\mathrm{LC} 50(\mathrm{~A})$ & Mortality & $\begin{array}{l}\text { Thamnocephalus } \\
\text { platyurus }\end{array}$ & This study \\
\hline & $>200$ & $96 h-E C 50(A)$ & $\begin{array}{l}\text { Morphology } \\
\text { perturbation }\end{array}$ & Hydra attenuata & This study \\
\hline Fish/fish cells & $>249$ & $48 \mathrm{~h}-\mathrm{TEC}^{\mathrm{a}}(\mathrm{A})$ & Cytotoxicity & $\begin{array}{l}\text { Oncorhynchus } \\
\text { mykiss (primary } \\
\text { hepatocytes) }\end{array}$ & This study \\
\hline Bivalve cells & 7.98 & $24 \mathrm{~h}-\operatorname{TEC}^{\mathrm{e}}(\mathrm{A})$ & $\begin{array}{l}\text { Phagocytosis } \\
\text { inhibition }\end{array}$ & $\begin{array}{l}\text { Hemocytes of } \\
\text { Elliptio complanata } \\
\text { (bivalve) }\end{array}$ & $\begin{array}{l}\text { Gagné et } \\
\text { al., 2006b }\end{array}$ \\
\hline \multicolumn{6}{|l|}{ Oxytetracycline $^{\mathrm{C}}$} \\
\hline \multirow[t]{3}{*}{ Bacteria } & $\begin{array}{l}3.0 \text { to } \\
6.9 \\
\end{array}$ & $4 \mathrm{~h}-\mathrm{IC} 50(\mathrm{~A})$ & $\begin{array}{l}\text { Growth rate } \\
\text { inhibition }\end{array}$ & $\begin{array}{l}\text { Activated sludge } \\
\text { micro-organisms }\end{array}$ & $\begin{array}{l}\text { Christensen } \\
\text { et al., } 2006\end{array}$ \\
\hline & 66 & $\begin{array}{l}15-\min \text { IC50 } \\
(\mathrm{A})\end{array}$ & $\begin{array}{l}\text { Luminescence } \\
\text { inhibition }\end{array}$ & Vibrio fischeri & $\begin{array}{l}\text { Christensen } \\
\text { et al., } 2006\end{array}$ \\
\hline & 135.9 & $\begin{array}{l}15-\min \text { IC50 } \\
\text { (A) }\end{array}$ & $\begin{array}{l}\text { Luminescence } \\
\text { inhibition }\end{array}$ & Vibrio fischeri & This study \\
\hline
\end{tabular}


Blaise et al.: Ecotoxicity of pharmaceuticals of urban origin.

Table 3 - (continuation)

\begin{tabular}{|c|c|c|c|c|c|}
\hline Substance & $\begin{array}{l}\text { Reported } \\
\text { value } \\
\text { (mg/L) }\end{array}$ & $\begin{array}{l}\text { Measurement } \\
\text { endpoint }\end{array}$ & $\begin{array}{l}\text { Assessment } \\
\text { endpoint }\end{array}$ & Species & Reference \\
\hline \multirow[t]{9}{*}{ Algae } & 0.2 & 72h-IC50 (C) & $\begin{array}{l}\text { Growth } \\
\text { inhibition }\end{array}$ & $\begin{array}{l}\text { Pseudokirchneriella } \\
\text { subcapitata }\end{array}$ & This study \\
\hline & 0.207 & $7 d-I C 50(C)$ & $\begin{array}{l}\text { Growth } \\
\text { inhibition }\end{array}$ & $\begin{array}{l}\text { Microcystis } \\
\text { aeruginosa } \\
\text { (cyanobacterium) }\end{array}$ & $\begin{array}{l}\text { Holten- } \\
\text { Lützhoft et al., } \\
1999\end{array}$ \\
\hline & 0.342 & $72 \mathrm{~h}-\mathrm{IC} 50(\mathrm{C})$ & $\begin{array}{l}\text { Growth } \\
\text { inhibition }\end{array}$ & $\begin{array}{l}\text { Pseudokirchneriella } \\
\text { subcapitata }\end{array}$ & $\begin{array}{l}\text { Eguchi et al., } \\
2004\end{array}$ \\
\hline & $\begin{array}{l}0.47 \text { to } \\
2.0\end{array}$ & 48h-IC50 (C) & $\begin{array}{l}\text { Growth rate } \\
\text { inhibition }\end{array}$ & $\begin{array}{l}\text { Pseudokirchneriella } \\
\text { subcapitata }\end{array}$ & $\begin{array}{l}\text { Christensen } \\
\text { et al., } 2006\end{array}$ \\
\hline & 4.18 & 72h-IC50 (C) & $\begin{array}{l}\text { Growth } \\
\text { inhibition }\end{array}$ & $\begin{array}{l}\text { Pseudokirchneriella } \\
\text { subcapitata }\end{array}$ & $\begin{array}{l}\text { De Liguoro et } \\
\text { al., } 2003\end{array}$ \\
\hline & 4.5 & 72h-IC50 (C) & $\begin{array}{l}\text { Growth } \\
\text { inhibition }\end{array}$ & $\begin{array}{l}\text { Pseudokirchneriella } \\
\text { subcapitata }\end{array}$ & $\begin{array}{l}\text { Holten- } \\
\text { Lützhoft et al., } \\
1999\end{array}$ \\
\hline & 5 & 72h-IC50 (C) & $\begin{array}{l}\text { Growth } \\
\text { inhibition }\end{array}$ & $\begin{array}{l}\text { Pseudokirchneriella } \\
\text { subcapitata }\end{array}$ & Webb, 2004 \\
\hline & 6.4 & 48h-IC50 (C) & $\begin{array}{l}\text { Growth } \\
\text { inhibition }\end{array}$ & Chlorella vulgaris & $\begin{array}{l}\text { Pro et al., } \\
2003\end{array}$ \\
\hline & 7.05 & $72 \mathrm{~h}-\mathrm{IC} 50$ (C) & $\begin{array}{l}\text { Growth } \\
\text { inhibition }\end{array}$ & Chlorella vulgaris & $\begin{array}{l}\text { Eguchi et al., } \\
2004\end{array}$ \\
\hline \multirow[t]{3}{*}{ Macrophytes } & 0.79 & 7d-EC10 (C) & $\begin{array}{l}\text { Growth } \\
\text { inhibition }\end{array}$ & Lemna gibba & $\begin{array}{l}\text { Brain et al., } \\
2004\end{array}$ \\
\hline & 1.01 & $7 d-I C 50(C)$ & $\begin{array}{l}\text { Growth } \\
\text { inhibition }\end{array}$ & Lemna gibba & $\begin{array}{l}\text { Brain et al., } \\
2004\end{array}$ \\
\hline & 4.92 & 7d-IC50 (C) & $\begin{array}{l}\text { Growth } \\
\text { inhibition }\end{array}$ & Lemna minor & $\begin{array}{l}\text { Pro et al., } \\
2003\end{array}$ \\
\hline \multirow[t]{4}{*}{$\begin{array}{l}\text { Micro- } \\
\text { invertebrates }\end{array}$} & 41.5 & 96h-EC50 (A) & $\begin{array}{l}\text { Morphology } \\
\text { perturbation }\end{array}$ & Hydra attenuata & This study \\
\hline & 46.2 & 21d-EC50 (C) & $\begin{array}{l}\text { Reproduction } \\
\text { inhibition }\end{array}$ & Daphnia magna & $\begin{array}{l}\text { Wollenberger } \\
\text { et al., } 2000\end{array}$ \\
\hline & 100 & 48h-EC10 (A) & Immobilization & Daphnia magna & $\begin{array}{l}\text { Wollenberger } \\
\text { et al., } 2000\end{array}$ \\
\hline & 337.6 & 24h-LC50 (A) & Mortality & $\begin{array}{l}\text { Thamnocephalus } \\
\text { platyurus }\end{array}$ & This study \\
\hline \multirow[t]{4}{*}{$\begin{array}{l}\text { Fish/fish } \\
\text { cells }\end{array}$} & 200 & 96h-LC50 (A) & Mortality & $\begin{array}{l}\text { Salvelinus } \\
\text { namaycush (Lake } \\
\text { trout) }\end{array}$ & Webb, 2004 \\
\hline & $>200$ & 24h-LC50 (A) & Cytotoxicity & $\begin{array}{l}\text { RTG-2 (rainbow } \\
\text { trout gonad cells) }\end{array}$ & $\begin{array}{l}\text { Babin et al., } \\
2005\end{array}$ \\
\hline & $>200$ & 24h-LC50 (A) & Cytotoxicity & $\begin{array}{l}\text { RTL-W1 (rainbow } \\
\text { trout liver cells) }\end{array}$ & $\begin{array}{l}\text { Babin et al., } \\
2005\end{array}$ \\
\hline & $>497$ & 48h-TEC ${ }^{a}(\mathrm{~A})$ & Cytotoxicity & $\begin{array}{l}\text { Oncorhynchus } \\
\text { mykiss (primary } \\
\text { hepatocytes) }\end{array}$ & This study \\
\hline \multicolumn{6}{|l|}{ Novobiocin } \\
\hline \multirow[t]{3}{*}{ Bacteria } & 0.08 & $5 h-I C 50(A)$ & $\begin{array}{l}\text { Growth } \\
\text { inhibition }\end{array}$ & Vibrio harveyi & $\begin{array}{l}\text { Thomulka et } \\
\text { al., } 1993\end{array}$ \\
\hline & 30.1 & $\begin{array}{l}\text { 15-min IC50 } \\
\text { (A) }\end{array}$ & $\begin{array}{l}\text { Luminescence } \\
\text { inhibition }\end{array}$ & Vibrio fischeri & This study \\
\hline & $>100$ & $1 \mathrm{~h}-\mathrm{IC} 50(\mathrm{~A})$ & $\begin{array}{l}\text { Luminescence } \\
\text { inhibition }\end{array}$ & Vibrio harveyi & $\begin{array}{l}\text { Thomulka et } \\
\text { al., } 1993\end{array}$ \\
\hline Algae & 130 & $\begin{array}{l}72 \mathrm{~h}-\mathrm{IC} 50 \\
(\mathrm{C})\end{array}$ & $\begin{array}{l}\text { Growth } \\
\text { inhibition }\end{array}$ & $\begin{array}{l}\text { Pseudokirchneriella } \\
\text { subcapitata }\end{array}$ & This study \\
\hline \multirow[t]{2}{*}{$\begin{array}{l}\text { Micro- } \\
\text { invertebrates }\end{array}$} & 135.2 & $\begin{array}{l}\text { 96h- } \\
\text { EC50 (A) }\end{array}$ & $\begin{array}{l}\text { Morphology } \\
\text { perturbation }\end{array}$ & Hydra attenuata & This study \\
\hline & 168.1 & $\begin{array}{l}24 \mathrm{~h}- \\
\text { LC50 (A) }\end{array}$ & Mortality & $\begin{array}{l}\text { Thamnocephalus } \\
\text { platyurus }\end{array}$ & This study \\
\hline
\end{tabular}


Braz. J. Aquat. Sci. Technol., 2006, 10(2):29-51.

Table 3 - (continuation)

\begin{tabular}{|c|c|c|c|c|c|}
\hline Substance & $\begin{array}{l}\text { Reported } \\
\text { value } \\
\text { (mq/L) }\end{array}$ & $\begin{array}{l}\text { Measurement } \\
\text { endpoint }\end{array}$ & $\begin{array}{l}\text { Assessment } \\
\text { endpoint }\end{array}$ & Species & Reference \\
\hline Fish/fish cells & 34.9 & $48 \mathrm{~h}-\mathrm{TEC}^{\mathrm{a}}(\mathrm{A})$ & Cytotoxicity & $\begin{array}{l}\text { Oncorhynchus } \\
\text { mykiss (primary } \\
\text { hepatocytes) }\end{array}$ & This study \\
\hline Bivalve cells & 0.635 & $24 \mathrm{~h}-\operatorname{TEC}^{\mathrm{e}}(\mathrm{A})$ & $\begin{array}{l}\text { Phagocytosis } \\
\text { inhibition }\end{array}$ & $\begin{array}{l}\text { Hemocytes of } \\
\text { Elliptio complanata } \\
\text { (bivalve) }\end{array}$ & $\begin{array}{l}\text { Gagné et } \\
\text { al., 2006b }\end{array}$ \\
\hline \multicolumn{6}{|l|}{ Trimethoprim } \\
\hline Bacteria & 183.3 & $\begin{array}{l}\text { 15-min IC50 } \\
\text { (A) }\end{array}$ & $\begin{array}{l}\text { Luminescence } \\
\text { inhibition }\end{array}$ & Vibrio fischeri & This study \\
\hline \multirow[t]{5}{*}{ Algae } & 80.3 & $72 \mathrm{~h}-\mathrm{IC} 50(\mathrm{C})$ & $\begin{array}{l}\text { Growth } \\
\text { inhibition }\end{array}$ & $\begin{array}{l}\text { Pseudokirchneriella } \\
\text { subcapitata }\end{array}$ & $\begin{array}{l}\text { Eguchi et } \\
\text { al., } 2004\end{array}$ \\
\hline & 96.7 & $72 \mathrm{~h}-\mathrm{IC} 50(\mathrm{C})$ & $\begin{array}{l}\text { Growth } \\
\text { inhibition }\end{array}$ & $\begin{array}{l}\text { Pseudokirchneriella } \\
\text { subcapitata }\end{array}$ & This study \\
\hline & 110 & $72 \mathrm{~h}-\mathrm{IC} 50(\mathrm{C})$ & $\begin{array}{l}\text { Growth } \\
\text { inhibition }\end{array}$ & $\begin{array}{l}\text { Pseudokirchneriella } \\
\text { subcapitata }\end{array}$ & $\begin{array}{l}\text { Halling- } \\
\text { Sorensen et } \\
\text { al. } 2000\end{array}$ \\
\hline & 112 & $7 d-I C 50(C)$ & $\begin{array}{l}\text { Growth } \\
\text { inhibition }\end{array}$ & $\begin{array}{l}\text { Microcystis } \\
\text { aeruginosa } \\
\text { (cyanobacterium) }\end{array}$ & $\begin{array}{l}\text { Holten- } \\
\text { Lützhoft et } \\
\text { al., } 1999\end{array}$ \\
\hline & 130 & $72 \mathrm{~h}-\mathrm{IC} 50(\mathrm{C})$ & $\begin{array}{l}\text { Growth } \\
\text { inhibition }\end{array}$ & $\begin{array}{l}\text { Pseudokirchneriella } \\
\text { subcapitata }\end{array}$ & $\begin{array}{l}\text { Holten- } \\
\text { Lützhoft et } \\
\text { al., } 1999\end{array}$ \\
\hline Macrophytes & $>1$ & 7-d EC10 (C) & $\begin{array}{l}\text { Growth } \\
\text { inhibition }\end{array}$ & Lemna gibba & $\begin{array}{l}\text { Brain et al., } \\
2004\end{array}$ \\
\hline \multirow[t]{3}{*}{$\begin{array}{l}\text { Micro- } \\
\text { invertebrates }\end{array}$} & $>85.3$ & 96h-EC50 (A) & $\begin{array}{l}\text { Morphology } \\
\text { perturbation }\end{array}$ & Hydra attenuata & This study \\
\hline & 123 & 48h-EC50 (A) & Immobilization & Daphnia magna & $\begin{array}{l}\text { Halling- } \\
\text { Sorensen et } \\
\text { al., } 2000\end{array}$ \\
\hline & 161.2 & 24h-LC50 (A) & Mortality & $\begin{array}{l}\text { Thamnocephalus } \\
\text { platyurus }\end{array}$ & This study \\
\hline \multirow[t]{2}{*}{ Fish/fish cells } & $>290$ & $48 \mathrm{~h}-\mathrm{TEC}^{\mathrm{a}}(\mathrm{A})$ & Cytotoxicity & $\begin{array}{l}\text { Oncorhynchus } \\
\text { mykiss (primary } \\
\text { hepatocytes) }\end{array}$ & This study \\
\hline & $>100$ & 72h-LC50 (A) & Mortality & Brachydanio rerio & $\begin{array}{l}\text { Halling- } \\
\text { Sorensen et } \\
\text { al., } 2000\end{array}$ \\
\hline \multicolumn{6}{|l|}{ Caffeine $^{\mathrm{d}}$} \\
\hline \multirow[t]{3}{*}{ Bacteria } & 569 & $\begin{array}{l}\text { 15-min IC50 } \\
\text { (A) }\end{array}$ & $\begin{array}{l}\text { Luminescence } \\
\text { inhibition }\end{array}$ & Vibrio fischeri & This study \\
\hline & 920 & $\begin{array}{l}24 \mathrm{~h}-\mathrm{MTC}^{*}(\mathrm{~A}) \\
{ }^{*} \text { microbial } \\
\text { toxic } \\
\text { concentration }\end{array}$ & $\begin{array}{l}\text { Growth } \\
\text { inhibition }\end{array}$ & $\begin{array}{l}\text { This assay uses an } \\
\text { array of } 11 \text { microbial } \\
\text { strains }\end{array}$ & $\begin{array}{l}\text { Gabrielson } \\
\text { et al., } 2003\end{array}$ \\
\hline & 2100 & $\begin{array}{l}\text { 15-min IC50 } \\
(\mathrm{A})\end{array}$ & $\begin{array}{l}\text { Luminescence } \\
\text { inhibition }\end{array}$ & Vibrio fischeri & $\begin{array}{l}\text { Ekwal et al., } \\
1998\end{array}$ \\
\hline Algae & 339.3 & $72 \mathrm{~h}-\mathrm{IC} 50(\mathrm{C})$ & $\begin{array}{l}\text { Growth } \\
\text { inhibition }\end{array}$ & $\begin{array}{l}\text { Pseudokirchneriella } \\
\text { subcapitata }\end{array}$ & This study \\
\hline Macrophytes & $>1$ & $7 \mathrm{~d}-\mathrm{EC} 10(\mathrm{C})$ & $\begin{array}{l}\text { Growth } \\
\text { inhibition }\end{array}$ & Lemna gibba & $\begin{array}{l}\text { Brain et al., } \\
2004\end{array}$ \\
\hline
\end{tabular}


Blaise et al.: Ecotoxicity of pharmaceuticals of urban origin.

Table 3 - (continuation).

\begin{tabular}{|c|c|c|c|c|c|}
\hline Substance & $\begin{array}{l}\text { Reported } \\
\text { value } \\
\text { (mg/L) }\end{array}$ & $\begin{array}{l}\text { Measurement } \\
\text { endpoint }\end{array}$ & $\begin{array}{l}\text { Assessment } \\
\text { endpoint }\end{array}$ & Species & Reference \\
\hline \multirow[t]{6}{*}{$\begin{array}{l}\text { Micro- } \\
\text { invertebrates }\end{array}$} & 160 & 48h-EC50 (A) & Immobilization & Daphnia magna & $\begin{array}{l}\text { Ekwal et al., } \\
1998\end{array}$ \\
\hline & $>200$ & $24 \mathrm{~h}-\mathrm{LC} 50(\mathrm{~A})$ & Mortality & $\begin{array}{l}\text { Thamnocephalus } \\
\text { platyurus }\end{array}$ & $\begin{array}{l}\text { Nalecz- } \\
\text { Jawecki } \\
\text { and } \\
\text { Persoone, } \\
2006\end{array}$ \\
\hline & 530.3 & $96 h-E C 50(A)$ & $\begin{array}{l}\text { Morphology } \\
\text { perturbation }\end{array}$ & Hydra attenuata & This study \\
\hline & 684 & $24 h-E C 50(A)$ & Immobilization & Daphnia magna & $\begin{array}{l}\text { Lilius et al., } \\
1994\end{array}$ \\
\hline & $>1500$ & $24 \mathrm{~h}-\mathrm{LC} 50(\mathrm{~A})$ & Mortality & $\begin{array}{l}\text { Thamnocephalus } \\
\text { platyurus }\end{array}$ & This study \\
\hline & 4661 & $24 h-L C 50(A)$ & Mortality & $\begin{array}{l}\text { Brachionus } \\
\text { calyciflorus }\end{array}$ & $\begin{array}{l}\text { Calleja et } \\
\text { al., } 1994\end{array}$ \\
\hline \multirow[t]{4}{*}{ Fish/fish cells } & 151 & 96h-EC50 (A) & Mortality & $\begin{array}{l}\text { Pimephales } \\
\text { promelas (fathead } \\
\text { minnow) }\end{array}$ & $\begin{array}{l}\text { Russom et } \\
\text { al., } 1997\end{array}$ \\
\hline & $>194$ & $48 \mathrm{~h}-\mathrm{TEC}^{\mathrm{a}}(\mathrm{A})$ & Cytotoxicity & $\begin{array}{l}\text { Oncorhynchus } \\
\text { mykiss (primary } \\
\text { hepatocytes) }\end{array}$ & This study \\
\hline & $>485$ & 24h-EC50 A) & Cytotoxicity & $\begin{array}{l}\text { PLHC-1 } \\
\text { (Poeciliopsis lucida) } \\
\text { hepatoma cells }\end{array}$ & $\begin{array}{l}\text { Caminada } \\
\text { et al., } 2006\end{array}$ \\
\hline & $>485$ & 24h-EC50 (A) & Cytotoxicity & $\begin{array}{l}\text { RTG-2 (rainbow } \\
\text { trout) cells }\end{array}$ & $\begin{array}{l}\text { Caminada } \\
\text { et al., } 2006\end{array}$ \\
\hline \multicolumn{6}{|l|}{ Cotinine } \\
\hline Bacteria & 369.3 & $\begin{array}{l}\text { 15-min IC50 } \\
\text { (A) }\end{array}$ & $\begin{array}{l}\text { Luminescence } \\
\text { inhibition }\end{array}$ & Vibrio fischeri & This study \\
\hline Algae & 589.5 & $72 \mathrm{~h}-\mathrm{IC} 50(\mathrm{C})$ & $\begin{array}{l}\text { Growth } \\
\text { inhibition }\end{array}$ & $\begin{array}{l}\text { Pseudokirchneriella } \\
\text { subcapitata }\end{array}$ & This study \\
\hline \multirow[t]{2}{*}{$\begin{array}{l}\text { Micro- } \\
\text { invertebrates }\end{array}$} & $>375$ & $96 h-E C 50(A)$ & $\begin{array}{l}\text { Morphology } \\
\text { perturbation }\end{array}$ & Hydra attenuata & This study \\
\hline & $>700$ & 24h-LC50 (A) & Mortality & $\begin{array}{l}\text { Thamnocephalus } \\
\text { platyurus }\end{array}$ & This study \\
\hline Fish/fish cells & 88.1 & $48 \mathrm{~h}-\mathrm{TEC}^{\mathrm{a}}(\mathrm{A})$ & Cytotoxicity & $\begin{array}{l}\text { Oncorhynchus } \\
\text { mykiss (primary } \\
\text { hepatocytes) }\end{array}$ & This study \\
\hline
\end{tabular}

a) Rainbow trout primary hepatocytes.

b) Threshold Effect Concentration (see Table 1, footnote b).

c) SOS Chromotest positive indicating DNA damage (without S9 mix) at a threshold effect concentration of $22.1 \mathrm{mg} / \mathrm{L}$.

d) SOS Chromotest positive indicating DNA damage (without S9 mix) at a threshold effect concentration of $354 \mathrm{mg} / \mathrm{L}$.

e) TEC (threshold effect concentration) for phagocytosis as manifested by a significant reduction in internalization capacity of fluorescent bacteria $=(\text { NOEC } x \text { LOEC })^{1 / 2}$, where NOEC $=$ no observed effect concentration and LOEC $=$ lowest observed effect concentration.

While caffeine has been placed in the «toxic» category based on an indeterminate value of $>1 \mathrm{mg} / \mathrm{L}$ reported for the macrophyte L. gibba (Table 3 ), this may be an unrealistic overestimate of its toxic potential and the real value may in fact lie in the «harmful» or «not toxic» categories. Other than toxicity data generated in our study for the nicotine metabolite cotinine (Table 3), none were found in the literature. In contrast, the parent molecule nicotine appears generally more toxic judging from aquatic toxicity values reported elsewhere : fathead minnow 96h-LC50 = 13.8 mg/L (Russom et al., 1997); fathead minnow $96 \mathrm{~h}-\mathrm{LC} 50=19.7 \mathrm{mg} / \mathrm{L}$; goldfish $96 \mathrm{~h}-$ 
$\mathrm{LC} 50=13.1 \mathrm{mg} / \mathrm{L} ;$ rainbow trout $96 \mathrm{~h}-\mathrm{LC} 50=7.31 \mathrm{mg} /$ $\mathrm{L}$; bluegill $96 \mathrm{~h}-\mathrm{LC} 50=4.31 \mathrm{mg} / \mathrm{L} ;$ D. magna $48 \mathrm{~h}-\mathrm{EC} 50$ $=3.25 \mathrm{mg} / \mathrm{L}$ (Holcombe et al., 1987); rainbow trout yolksac stage post-hatch fry $96 \mathrm{~h}-\mathrm{LC} 50=4 \mathrm{mg} / \mathrm{L}$ (Edsall, 1991); D. pulex $48 \mathrm{~h}-\mathrm{EC} 50=0.24 \mathrm{mg} / \mathrm{L}$ (Smith et al., 1988). After metabolic transformation, toxic potency therefore decreases from «very toxic» (nicotine) to one of «harmful» or «not toxic» (cotinine), based on EUDirective classification (Table 4).

The anti-inflammatory agents (ibuprofen, naproxen) and lipid regulators (gemfibrozil, bezafibrate) display a somewhat similar risk profile ranging from «very toxic» to «not toxic». Algae, cyanophytes, invertebrates and macrophytes appear particularly more sensitive to these classes of pharmaceuticals than other groups owing to their responses in the "very toxic» and «toxic» class ranges (Table 4). Toxic responses for the anticonvulsant carbamazepine are surprisingly homogeneous as they all fall in the «harmful» category, thereby suggesting a lower risk for aquatic life than that of other groups of substances. Acute toxicity results on the ecotoxicity of pharmaceuticals also show reported effects for carbamazepine ranging from $12.7 \mathrm{mg} / \mathrm{L}$ and higher (Fent et al., 2006).

The spectrum of toxicity responses for the five antibiotics is the most diversified ranging from «extremely toxic» to «not toxic» (Table 4). More susceptible to their action are algae, bacteria, bivalve cells, cyanophytes and macrophytes whereas invertebrates and fish/fish cells appear less sensitive. For example, sulfamethoxazole is shown to be «extremely toxic» to the cyanophyte $S$. leopolensis and, likewise, novobiocin to the luminescent bacterium $V$. harveyi (Tables 3 and 4). Respective exposure of these two micro-organisms to sulfamethoxazole $(96 \mathrm{~h}-\mathrm{IC} 50=0.027 \mathrm{mg} / \mathrm{L})$ and novobiocin $(0.08 \mathrm{mg} / \mathrm{L})$ resulted in the highest toxicity values reported in Table 3. Again, sulfamethoxazole, novobiocin, oxytetracycline and sulfapyridine produce "very toxic» and «toxic» responses in algae, bacteria, bivalve cells or macrophytes. Of the five antibiotics, trimethoprim appears less bioactive as it proved to be possibly «toxic» to macrophytes, «harmful» to algae and «not toxic» to all other taxonomic groups (Table 4).

Overall, fish/fish cells were the least sensitive of all biotic groups when challenged with the 12 pharmaceuticals. Barring a «harmful» hazard potency for carbamazepine and cotinine, all other responses fell in the «not toxic» category (Table 4). An acute toxicity data base constructed for over 100 pharmaceuticals showed that fish were less sensitive than algae and invertebrates (Webb, 2004). The apparent lesser sensitivity of fish to pharmaceuticals was also pointed

Table 4 - Toxicity of the pharmaceutical groups found in the municipal wastewater treatment plant effluent according to European Union Commission Guideline 93/67/EEC classification and based on measurement endpoint values (LC50, EC50, IC50, threshold effect concentration, etc.) reported for the most sensitive bioassay listed in Table 3.

\begin{tabular}{|c|c|c|c|c|c|}
\hline Group & $\begin{array}{c}\text { Extremely toxic } \\
(<0.1 \mathrm{mg} / \mathrm{L})\end{array}$ & $\begin{array}{c}\text { Very toxic } \\
(0.1-1 \mathrm{mg} / \mathrm{L})\end{array}$ & $\begin{array}{c}\text { Toxic } \\
(1-10 \mathrm{mg} / \mathrm{L}) \\
\end{array}$ & $\begin{array}{c}\text { Harmful } \\
(10-100 \mathrm{mg} / \mathrm{L}) \\
\end{array}$ & $\begin{array}{c}\text { Not toxic } \\
(>100 \mathrm{mg} / \mathrm{L})\end{array}$ \\
\hline $\begin{array}{l}\text { Anti-inflammatory agents : ibuprofen } \\
\text { (ibu), naproxen (nap) }\end{array}$ & & $\begin{array}{l}\text { C-ibu } \\
\text { I-nap } \\
\text { M-nap }\end{array}$ & M-ibu & $\begin{array}{l}\text { A-ibu/nap } \\
\text { B-ibu/nap }\end{array}$ & F-ibu/nap \\
\hline $\begin{array}{l}\text { Lipid regulators : gemfibrozil (gem), } \\
\text { bezafibrate (bez)) }\end{array}$ & & I-gem & $\begin{array}{l}\text { A-gem } \\
\text { l-gem/bez }\end{array}$ & $\begin{array}{l}(\mathrm{A}-\mathrm{bez})^{\mathrm{a}} \\
(\mathrm{B}-\mathrm{bez})^{\mathrm{a}}\end{array}$ & F-gem/bez \\
\hline Anti-convulsant : carbamazepine (cbz)) & & & $(\mathrm{M}-\mathrm{cbz})^{\mathrm{a}}$ & $\begin{array}{l}\text { A-cbz } \\
\text { B-cbz } \\
\text { C-cbz } \\
\text { F-cbz } \\
\text { I-cbz }\end{array}$ & \\
\hline $\begin{array}{l}\text { Antibiotics : sulfamethoxazole (smx), } \\
\text { sulfapyridine (spy), oxytetracycline (oxy), } \\
\text { novobiocin (nov), trimethoprim (tri) }\end{array}$ & B-nov C-smx & $\begin{array}{l}\text { A-smx/oxy } \\
\text { Bi-nov } \\
\text { C-oxy } \\
\text { M-oxy }\end{array}$ & $\begin{array}{l}\text { A-spy } \\
\text { B-oxy } \\
\text { Bi-spy } \\
\text { (M-tri) }\end{array}$ & $\begin{array}{l}\text { A-tri } \\
\text { B-smx } \\
\text { Bi-smx } \\
\text { I-smx/oxy }\end{array}$ & $\begin{array}{l}\text { A-nov } \\
\text { B-spy/tri } \\
\text { C-tri } \\
\text { F-smx/spy/oxy/nov/tri } \\
\text { l-spy/nov/tri }\end{array}$ \\
\hline Stimulant : caffeine (caf) & & & $(\mathrm{M}-\mathrm{caf})^{\mathrm{a}}$ & & $\begin{array}{l}\text { A-caf } \\
\text { B-caf } \\
\text { F-caf } \\
\text { l-caf }\end{array}$ \\
\hline Nicotine metabolite : cotinine (cot) & & & & $\mathrm{F}-\cot$ & $\begin{array}{l}\text { A-cot } \\
\text { B-cot } \\
\text { l-cot }\end{array}$ \\
\hline
\end{tabular}

Taxonomic groups : A (algae); B (bacteria); Bi (bivalve cells); C (cyanobacteria); F (fish/fish cells); I (invertebrates); M (macrophytes). a) Based on an inderminate value of "greater than» $(>)$ for this class (see Table 3 ).

b) Example : For antibiotics (oxytetracycline), the green alga $P$. subcapitata gave the most sensitive response for reported algal toxicity tests (IC50 = $0.2 \mathrm{mg} / \mathrm{L}$, Table 3$)$, thereby placing this compound in the «Very Toxic» category. 
out in a review undertaken by Carlsson et al. (2006) on the environmental risk assessment of 27 active pharmaceutical ingredients. The authors reported that acute toxicities of 24 of these were shown to differ markedly based on taxonomic group (micro-organisms, algae, invertebrates and fish) with algae being the most sensitive (effects at $\mu \mathrm{g} / \mathrm{L}$ for antibiotics) and fish generally the least sensitive (effects greater than $1000 \mathrm{mg} / \mathrm{L}$ for some pharmaceuticals). That fish appear less prone to the adverse effects of antibiotics was also noted by Crane et al. (2006). In their own review, Fent et al. (2006) state that fish were, in general, less sensitive to pharmaceuticals than other trophic levels.

Comparing the highest and lowest sensitivity responses of bioassay data reported in Table 3 for each pharmaceutical offers further insights regarding their toxicity (Table 5). Indeed, the toxic spread index (tsi) gives some information about whether toxicity is generic (low index value) or specific (high index value). On the one hand, cotinine, whose calculated tsi value is $>7.9$, is indicative of a narrow span of toxic effects towards all taxonomic groups. Sulfamethoxazole, on the other hand, whose tsi value is $>9370$, indicates toxic effects that are more or less intense depending on the exposed biotic group. Cotinine's low tsi value is also clearly reflected by its hazard potential essentially confined to the «not toxic» category, whereas sulfamethoxazole's high tsi value spreads across all categories of toxicity (Table 4). This suggests that pharmaceuticals with large tsi values are likely more hazardous to aquatic environments if they feature a broad range of effects on a large number of species across several biological levels. However, drugs having low tsi index values (as per Table 5) but categorized in the «toxic" to «extremeley toxic» categories (as per Table 4) would also present greater risk. For the non-antibiotics studied, cotinine, caffeine, bezafibrate and carbamazepine display lower tsi values than ibuprofen, naproxen and gemfibrozil. For the five antibiotics, tsi values for novobiocin, oxytetracycline and sulfamethoxazole are markedly above those of sulfapyridine and trimethoprim (Table 5). The most sensitive endpoint values reported in Table 5 are also informative about pharmaceutical concentrations at which the most susceptible species can be affected.

Table 5 - Toxic spread index (ratio of least sensitive endpoint value $\div$ most sensitive endpoint value) based on the comparative sensitivity responses of bioassays reported in Table 3 to the 12 pharmaceuticals.

\begin{tabular}{|c|c|}
\hline Pharmaceutical class & $\begin{array}{c}\text { Toxic spread } \\
\text { index }\end{array}$ \\
\hline \multicolumn{2}{|l|}{ Non-antibiotics } \\
\hline $\begin{array}{l}\text { Cotinine: }>700 \mathrm{mg} / \mathrm{L}(\text { T. platyurus }) \div 88.1 \mathrm{mg} / \mathrm{L} \text { (Fish primary } \\
\text { hepatocyte cells) }\end{array}$ & $>7.9$ \\
\hline Caffeine: $4661 \mathrm{mg} / \mathrm{L}$ (B. calyciflorus) $\div 151 \mathrm{mg} / \mathrm{L}$ (P. promelas) & 30.9 \\
\hline $\begin{array}{l}\text { Bezafibrate: } 941 \mathrm{mg} / \mathrm{L} \text { (Fish PLHC-1 cells) } \div 5.3 \mathrm{mg} / \mathrm{L}(H \text {. } \\
\text { attenuata) }\end{array}$ & 178 \\
\hline $\begin{array}{l}\text { Carbamazepine: }>591 \text { mg/L (Fish RTG-2 and PLHC-1 cells) } \div>1 \\
\text { mg/L }(\text { L. gibba })^{\mathrm{a}}\end{array}$ & $>296$ \\
\hline $\begin{array}{l}\text { lbuprofen: } 1194 \text { mg/L (Fish RTG -2 cells) } \div 1 \text { mg/L (Synechosystis } \\
\text { sp.) }\end{array}$ & 1194 \\
\hline Naproxen: 1032 mg/L (Fish RTG-2 cells) $\div 0.33$ mg/L (C. dubia) & 1233 \\
\hline $\begin{array}{l}\text { Gemfibrozil: } 986 \text { mg/L (Fish RTG }-2 \text { cells }) \div 0.8 \text { mg/L }(H \text {. } \\
\text { attenuata) }\end{array}$ & 3127 \\
\hline \multicolumn{2}{|l|}{ Antibiotics } \\
\hline $\begin{array}{l}\text { Sulfapyridine: }>249 \mathrm{mg} / \mathrm{L} \text { (Fish primary hepatocyte cells) } \div 7.98 \\
\mathrm{mg} / \mathrm{L} \text { (Bivalve cells) }\end{array}$ & $>31.2$ \\
\hline $\begin{array}{l}\text { Trimethoprim: }>290 \mathrm{mg} / \mathrm{L} \text { (Fish primary hepatocyte cells }) \div>1 \\
\mathrm{mg} / \mathrm{L}(\text { L. gibba })^{\mathrm{a}}\end{array}$ & $>145$ \\
\hline Novobiocin: $168.1 \mathrm{mg} / \mathrm{L}$ (T. platyurus) $\div 0.08 \mathrm{mg} / \mathrm{L}(\mathrm{V}$. harveyi) & 2101 \\
\hline $\begin{array}{l}\text { Oxytetracycline: }>497 \mathrm{mg} / \mathrm{L} \text { (Fish primary hepatocyte cells) } \div 0.2 \\
\text { mg/L ( } P \text {. subcapitata })\end{array}$ & $>2485$ \\
\hline $\begin{array}{l}\text { Sulfamethoxazole: }>253 \mathrm{mg} / \mathrm{L} \text { (Fish primary hepatocyte cells) } \div \\
0.027 \mathrm{mg} / \mathrm{L}(\mathrm{S} \text {. leopolensis) }\end{array}$ & $>9370$ \\
\hline
\end{tabular}

a) The value of ">1 mg/L" reported for Lemna gibba by Brain et al., (2004) in Table 3, their highest experimental concentration, was set at "2" for the purpose of this exercise.

b) The value of " $>0.01 \mathrm{mg} / \mathrm{L}$ " reported for Hydra vulgaris by Pascoe et al., (2003) in Table 3, their highest experimental concentration, was not considered for the purpose of this exercise. 
Notably, and depending on the substance being considered, one species of each of the taxonomic groups tested was always found to be the most sensitive. This pleads in favor of applying future test batteries for toxicity assessment of existing and new pharmaceuticals that should incorporate sensitive species from representative biological levels so as to circumscribe their full ecotoxic potential. Based on our study of 12 substances that encompass several classes of pharmaceuticals (Table 2), the alga $P$. subcapitata, the bacterium $V$. harveyi, the cyanophytes Synechosystis sp. and S. leopolensis, the invertebrates $C$. dubia and $H$. attenuata, as well as the macrophyte L. gibba, all appear to be good candidates for this purpose (Table 5).

The relationship between bioassay effect concentrations for the pharmaceuticals investigated and those found in WWTPs offers notions about their risk potential towards aquatic biota (Fig. 1). Based on maximal concentrations of pharmaceuticals reported in either the Montreal WWTP or those reported elsewhere, the most sensitive bioassay response differs by anywhere from less than one to more than six orders of magnitude. Owing to their toxic effects determined at levels close to the range of wastewater concentrations, sulfamethoxazole, naproxen and ibuprofen would appear to pose greater risk to aquatic life, while less risk would be expected from oxytetracycline, gemfibrozil and novobiocin, whose biological effects are two orders of magnitude higher than concentrations emitted by WWTPs. The remaining compounds appear to pose little threat because of the wide gap separating their most sensitive bioassay response and reported WWTP concentrations (i.e., three to six orders of magnitude difference).

Beyond the unquestionable use of acute and chronic toxicity tests for screening of emerging chemicals such as pharmaceuticals, more subtle adverse effects possibly resulting from the biotransformation of drugs uptaken by biota deserve attention.

In particular, oxidative stress owing to the release of reactive oxygen species can be a consequence of metabolization following xenobiotic accumulation (Winston et al., 1996). In fact, acute exposure of the cnidarian Hydra attenuata to carbamazepine for $48 \mathrm{~h}$ induced oxidative metabolism at a threshold level of 7.1 $\mu \mathrm{g} / \mathrm{L}$ with a concomitant increase in lipid peroxidation (Quinn et al., 2004). This concentration is similar to the maximum value of $6.3 \mu \mathrm{g} / \mathrm{L}$ reported in WWTPs for this substance (Ternes, 1998), suggesting that oxidative

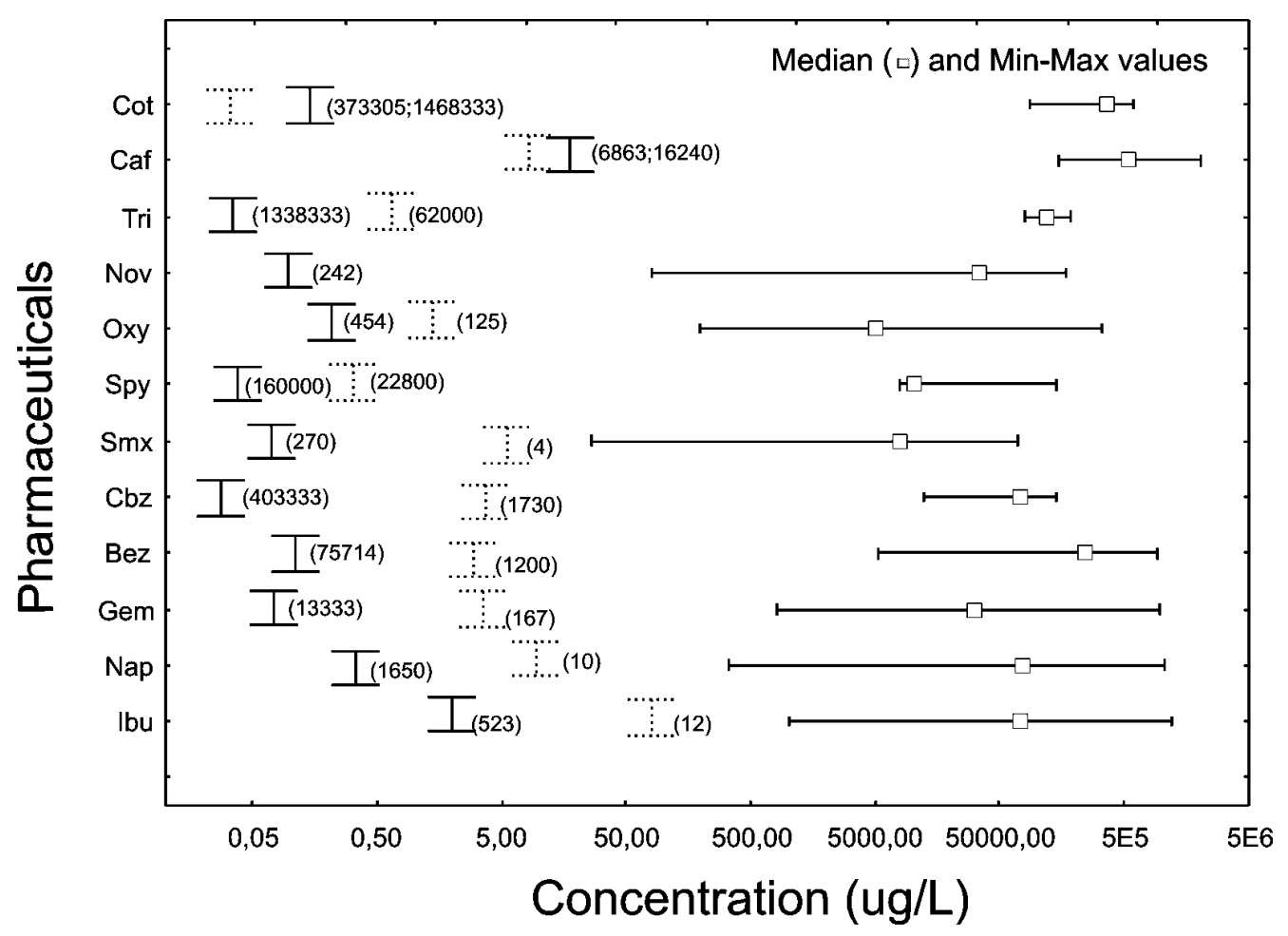

Figure 1 - Acute and chronic toxicity of the 12 pharmaceuticals (all biological levels confounded) and relationship with maximal concentrations found in WWTP effluents. Only determinate bioassay endpoint values in Table 3 were used to construct this scale. The vertical bars refer to maximal WWTP effluent concentrations found in the Montreal-based plant (full bars) and elsewhere (dotted bars). Values in parentheses are respective ratios of the maximum WWTP effluent concentration reported for each pharmaceutical and the corresponding most sensitive bioassay response obtained. 
stress could occur at levels close to those discharged by municipal effluents. Since the sensitivity of Hydra has been shown to increase 10-15 fold under conditions of chronic exposure (21 days versus 4 days) to toxic samples (Arkhipchuk et al., 2006), effect concentrations producing oxidative stress after metabolization of carbamazepine or other xenobiotics may even decrease further under situations of prolonged interaction.

Of the 12 pharmaceuticals studied, caffeine consistently yielded bioassay responses that were $>100$ $\mathrm{mg} / \mathrm{L}$ (Table 3 ) and was therefore classified as «not toxic» according to EU-Directive 93/67/EEC (Table 4). However, following a 48h exposure of caffeine to Hydra attenuata, a significant activity of xanthine oxydoreductase, indicative of oxidative biotransformation, was measured (Fig. 2). Since this enzyme transforms hypoxanthine (and caffeine) into uric acid and hydrogen peroxide, oxidative stress occurs and reactive oxygen species may cause cellular damage. Results show that xanthine oxidative metabolism is induced in Hydra at $=0.2 \mathrm{mg} / \mathrm{L}$ which is only 10 -fold the $0.022 \mathrm{mg} / \mathrm{L}$ concentration measured in the Montreal-based WWTP effluent (Table 2).

Since oxidative stress resulting from metabolization of uptaken drugs could realistically be manifest at even lower concentrations under scenarios of chronic or continuous exposure to caffeine and other pharmaceuticals, such effects certainly warrant future study. More subtle effects owing to uptake of pharmaceuticals have also been highlighted for gemfibrozil where exposure to environmental levels by Carassius auratus (goldfish) leads to bioconcentration of the drug in plasma with consequent endocrine disrupting effects evidenced by a decrease in plasma testosterone (Mimeault et al., 2005).

\section{CONCLUSIONS}

Chemical analysis of a Montreal-based WWTP effluent discharging to the Saint-Lawrence River identified the presence of 12 pharmaceuticals comprising antiinflammatory agents, lipid regulators, an anti-convulsant, antibiotics, a simulant and a nicotine metabolite. Bioanalytical assessment of these substances with our own suite of tests complemented by acute and chronic toxicity data reported in the literature confirmed that most of these substances can be classified as toxic according to EU-Directive 93/67/EEC, although their measured concentrations in WTTPs are essentially too

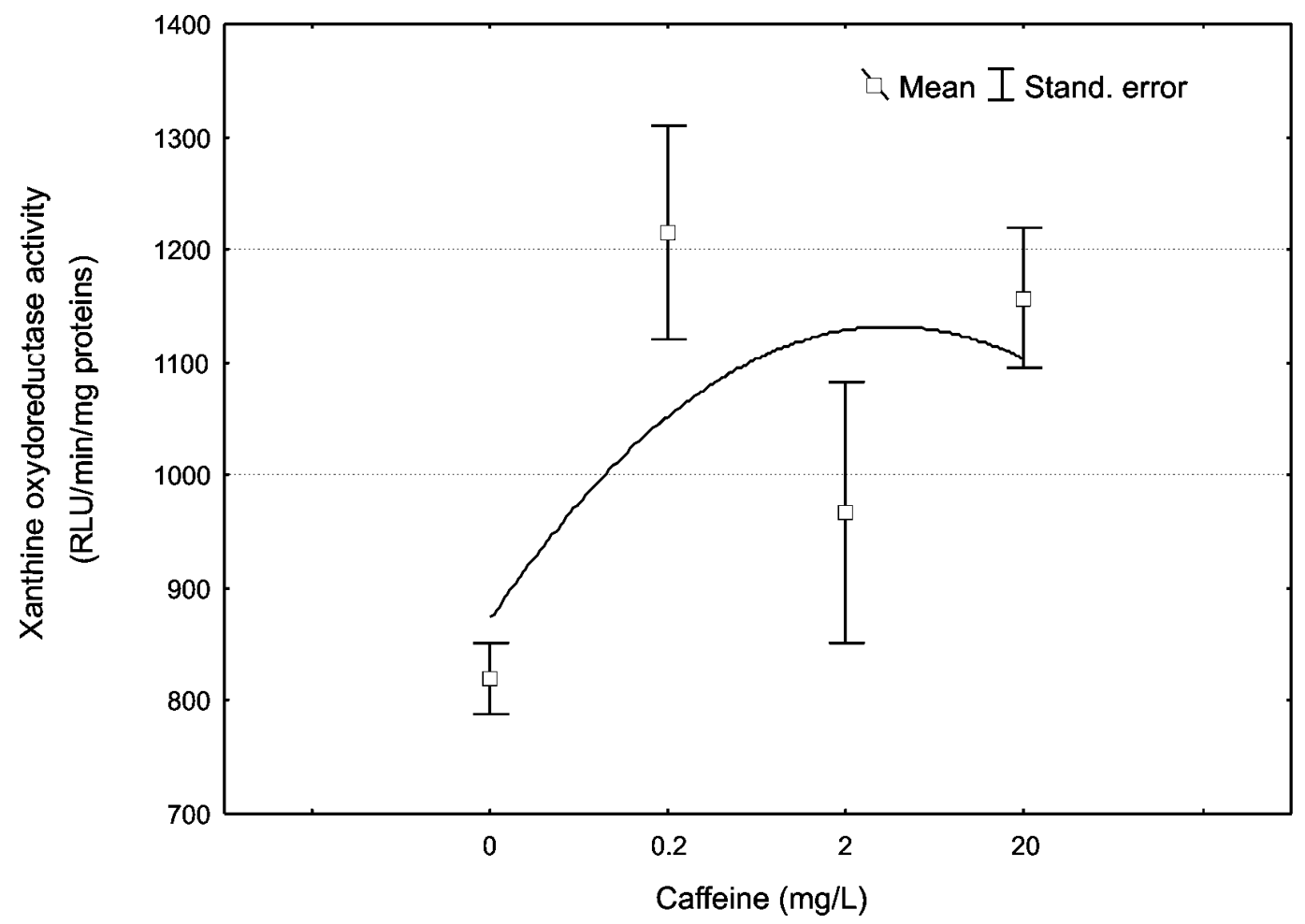

Figure 2 - Xanthine oxydoreductase activity after a 48h exposure of Hydra attenuata to caffeine. The data represent the mean with standard error from $\mathrm{N}=3$ replicates. Curve adjustment was performed using the exponential negative ponderation model (Statistica software, version 7.1). 
low to cause acute or chronic effects on an individual basis. Exceptions are perhaps sulfamethoxazole, naproxen and ibuprofen whose toxic effects were found to occur at concentrations close to those found in municipal effluents.

Overall, bioassay responses to the pharmaceuticals showed a wide range of sensitivity that advocates the use of tests representing diverse taxonomic levels to properly appraise their full toxic potential. Some species from each biotic group employed in toxicity testing were shown to be particularly sensitive toward individual drugs and certainly merit being part of future test batteries to evaluate other emerging substances such as pharmaceuticals. Beyond acute and chronic toxicity testing of pharmaceuticals, we intimated that metabolization of drugs uptaken by biota (e.g., induced activity of xanthine oxydoreductase in Hydra by caffeine, an essentially non toxic molecule) may lead to more insidious outcomes such as oxidative stress. These effects may be manifest at drug concentrations close to those present in urban effluents particularly under conditions of chronic or continuous exposure.

As recommended by others, we emphasize that future appraisal of the ecotoxicity of pharmaceuticals should continue to include bioassays (acute and chronic testing) of individual products and of mixtures (determination of interactive effects) at various trophic levels. Biomarker measurements are also required to assess the consequences associated with the biotransformation of drugs by biota. Fish, bivalves and other invertebrates, for example, are particularly suited for this purpose. Finally, recalcitrant drugs with lipophilic activity (e.g., $\mathrm{K}_{\mathrm{ow}} \geq 2$ ) need to be investigated to assess their potential to bio-accumulate and to bio-magnify up the food chain. We are presently developing simple experimental trophic chains in the laboratory for this goal.

\section{ACKNOWLEDGMENTS}

This work was conducted under the Municipal Effuent Research Program of Environment Canada, Aquatic Ecosystem Protection Research Division, Centre Saint-Laurent Research Group, with funding provided by the Saint-Lawrence River Action Plan. We are grateful to Pavleta Pavlota, student of Professor Robert Tardif, Department of Occupational and Environmental Health, University of Montréal, for her contribution to some of the literature review on the presence of the 12 pharmaceuticals in WWTPs and on their associated toxic effects. We also acknowledge Manon Harwood and Chantale André for their assistance in generating bioassay and biomarker data, respectively.
Effluent concentrations of pharmaceuticals in Table 2 are reprinted from Gagné et al., 2006a, Table 1, page 331 , with permission from Elsevier.

\section{REFERENCES}

Arkhipchuk, V.; Blaise, C. \& Malinovskaya, M. 2006. Use of Hydra for chronic toxicity assessment of waters intended for human consumption. Environ. Poll. 142: 200-211.

Andreozzi, R.; Raffaele, M., \& Nicklas, P. 2003. Pharmaceuticals in STP effluents and their solar photodegradation in aquatic environment. Chemosphere 50: 1319-1330.

Ashton, D.; Hilton, M. \& Thomas, K.V. 2004. Investigating the environmental transport of human pharmaceuticals to streams in the United Kingdom. Sci. Total Environ. 333: 167-184.

Babin, M.; Boleas, S. \& Tarazona, J.V. 2005. In vitro toxicity of antimicrobials on RTG-2 and RTL-W1 fish cell lines. Environ. Toxicol. Pharmacol. 20: 125-134.

Batt, A.L.; Bruce, I.B. \& Aga, D.S. 2006a. Evaluating the vulnerability of surface waters to antibiotic contamination from varying wastewater treatment plant discharges. Environ. Poll. 142: 295-302.

Batt, A.L.; Kim, S. \& Aga, D.S. 2006b. Enhanced biodegradation of ioprodine and trimethoprim in nitrifying activated sludge. Environ. Sci. Technol. 40: 7367-7373.

Blaise, C. \& Kusui, T. 1997. Acute toxicity assessment of industrial effluents with a microplate-based Hydra attenuata assay. Environ. Toxicol. Water Qual. 12: 53-60.

Blaise, C.; Trottier, S., Gagné, F., Lallement, C. \& Hansen, P.-D. 2002. Immunocompetence of bivalve hemocytes by a miniaturized phagocytosis assay. Environ. Toxicol. 17: 160-169.

Blaise, C.; Gagné, F., Salazar, M., Salazar, S., Trottier, S. \& Hansen, P.-D. 2003. Experimentally-induced feminisation of freshwater mussels after long-term exposure to a municipal effluent. Fresenius Environmental Bulletin 12: 865-870.

Blaise, C. \& Vasseur, P. 2005. Algal Microplate Toxicity test. In: Blaise, C. \& Férard, J.F. (eds.) Small-scale Freshwater Toxicity Investigations, Volume 1, Springer, Dordrecht, 137-179 pp.

Bradford, M.M., 1976. A rapid and sensitive method for the quantification of microgram quantities of protein utilizing the principle of protein-dye binding. Anal. Biochem. 72, 248-251.

Bowden, C.L. 1997. Choosing the appropriate therapy for bipolar disorder. Medscape Mental Health, 2( 8). 
Brain, R.A.; Johnson, D.J., Richards, S.M., Sanderson, H., Sibley, P.K. \& Solomon, K.R., 2004. Effects of 25 pharmaceutical compounds to Lemna gibba using a seven-day static renewal test. Environ. Toxicol. Chem. 23: 371-382.

Buerge, I.J.; Poiger, T., Müller, M.D. \& Buser, H-R. 2003. Caffeine, an anthropogenic marker for wastewater contamination of surface waters. Environ. Sci. Technol. 37: 691-700.

Buser,H.R.; Poiger, T. \& Müller, M.D. 1999. Occurrence and environmental behavior of pharmaceutical drug ibuprofen in surface waters and wastewater. Environ. Sci. Technol. 33: 2229-2535.

Calleja, M.C.; Persoone, G. \& Geladi, P. 1994. Comparative acute toxicity of the first 50 Multicentre Evaluation of in vitro cytotoxicity chemicals to aquatic non-vertebrates. Arch. Environ. Contam. Toxicol. 26: 69-78.

Caminada, D.; Escher, C. \& Fent, K. 2006. Cytotoxcicity of pharmaceuticals found in aquatic systems: comparison of PLHC-1 and RTG-2 fish cell lines. Aquat. Toxicol. 79: 114-123.

Carlsson, C.; Johansson, A.K., Alvan, G., Bergman, K. \& Kühler, T. 2006. Are pharmaceuticals potent environmental pollutants? Part 1: Environmental risk assessments of selected active pharmaceutical ingredients. Sci. Total Environ. 364: 67-87.

CEC (Commission of the European Communities), 1996. Technical guidance document in support of commission directive 93/67/EEC on risk assessment for new notified substances. Part II, Environmental Risk Assessment. Office for official publications of the European Communities, Luxembourg.

Christensen, A.M.; Ingerslev, F. \& Baun, A. 2006. Ecotoxicity of mixtures of antibiotics used in aquacultures. Environ. Toxicol. Chem. 8:2208-2215.

Cleuvers, M., 2003. Aquatic ecotoxicity of pharmaceuticals including the assessment of combination effects. Toxicol. Letters, 142: 185-194.

Cleuvers, M., 2004. Mixture toxicity of the antiinflammatory drugs diclofenac, ibuprofen, naproxen and acetylsalicylic acid. Ecotox. Environ. Safety 59: 309-315.

Crane, M.; Watts, C. \& Boucard, T. 2006. Chronic aquatic environmental risks from exposure to human pharmaceuticals. Sci. Total Environ. 367: 23-41.

Cunningham, V.L.; Buzby, M., Hutchinson, T., Mastrocco, F., Parke, N. \& Roden, N. 2006. Effects of Human Pharmaceuticals on Aquatic Life: Next Steps. Environ. Sci. Tech. 40: 3456-3462.

De Liguoro, M.; Cibin, V., Capolongo, F., HallingSorensen, B. \& Montesissa, C. 2003. Use of tetracycline and tylosin in intensive calf farming: evaluation of transfer to manure and soil. Chemosphere 52: 203-212.
Dietrich, D.L.; Webb, F. \& Petry, T. 2002. Hot spot pollutants: pharmaceuticals in the environment, Toxicol. Letters 131: 1-3.

Edsall, C.C. 1991. Acute toxicities to larval Rainbow Trout of representative compounds detected in Great Lakes fish. Bull. Environ. Contam. Toxicol. 46: 173178.

Eguchi, K.; Nagase, H., Ozawa, M., Endoh, Y.S., Goto, K., Hirata, K., Miyamoto, K. \& Yoshimura, H. 2004. Evaluation of antimicrobial agents for veterinary use in the ecotoxicity test using microalgae. Chemosphere 57: 1733-1738.

Ekwall, B.; Barile, F.A., Castano, A. Clemedson, C., Clothier, R.H., Dierickx, P., Ekwall, B., Ferro, M., Fiskesjo", G., Garza-Ocan as, L., Go'mez-Lecho'n, M.J., Gülden, M., Hall, T., Isomaa, B., Kahru, A., Kerszman, G., Kristen, U., Kunimoto, M., Ka"renlampi, S., Lewan, L., Loukianov, A., Ohno, T., Persoone, G., Romert, L., Sawyer, T.W., Shrivastava, R., Segner, H., Stammati, A., Tanaka, N., Valentino, M., Walum, E., Zucco, F. 1998. MEIC Evaluation of Acute Systemic Toxicity: Part VI. The prediction of human toxicity by rodent LD50 values and results from 61 in vitro methods. ATLA(Alternat. Lab. Animals) 26: 617-658.

Environment Canada. 1992. Biological test method: toxicity test using luminescent bacteria (Photobacterium phosphoreum). Environmental Protection Series, Report EPS 1/RM/24, Conservation and Protection, Environment Canada, Ottawa, 61 pages.

Environnement Canada et Ministère de l'Environnement et de la Faune du Québec, 1998. Evaluation de la toxicité des effluents des stations d'épuration municipales du Québec. Saint-Laurent Vision 2000, juillet 1998, 88 pages + annexes.

Fent, K.; Weston, A. \& Caminada, D. 2006. Ecotoxicology of human pharmaceuticals. Aquat. Toxicol. 76: 122-159.

Ferrari, B.; Mons, R., Vollat, B., Fraysse, B., Paxéus, N., Lo Giudice, R., Pollio, A. \& Garric, J. 2004. Environmental risk assessment of six human pharmaceuticals: are the current environmental risk assessment procedures sufficient for the protection of the aquatic environment? Environ. Toxicol. Chem. 23: $1344-1354$.

Flaherty, C.M. \& Dodson, S.I. 2005. Effects of pharmaceuticals on Daphnia survival, growth and reproduction. Chemosphere 61:200-207.

Gabrielson, J.; Kühn, I., Colque-Navarro, P., Hart, M., Iversen, A., McKenzie, D. \& Möllby, R. 2003. Microplate-based microbial assay for risk assessment and (eco)toxic fingerprinting of chemicals. Analytica Chimica Acta 485: 121-130. 
Gagné, F. 2005. Acute toxicity assessment of liquid samples with primary cultures of rainbow trout hepatocytes. In: Blaise, C. \& Férard, J.F. Smallscale Freshwater Toxicity Investigations, Volume 1, Springer, Dordrecht, 453-472 pp.

Gagné F.; Blaise C., Salazar M., Salazar S. \& Hansen, P.D. 2001. Evaluation of estrogenic effects of municipal effluents to the freshwater mussel Elliptio complanata. Comp. Biochem. Physiol. 128C: 213225.

Gagné, F.; Blaise, C. \& André, C. 2006a. Occurrence of pharmaceutical products in a municipal effluent and toxicity to rainbow trout (Oncorhynchus mykiss) hepatocytes. Ecotoxicol, Environ Saf. 64: 329-336.

Gagné, F.; Blaise, C., Fournier, M. \& Hansen, P.D. 2006b. Effects of selected pharmaceutical products on phagocytic activity of Elliptio complanata mussels. Comp. Biochem. Physiol. C 143: 179186.

Gagné, F.; Eullaffroy, P. \& Blaise, C. 2006c. Development of a competitive enzymatic immunoassay for carbamazepine in biological tissues. Fresenius Environ. Bull. 15: 1512-1516.

Garric, J. \& Ferrari, B. 2005. Les substances pharmaceutiques dans les milieux aquatiques. Niveaux d'exposition et effet biologique : que savonsnous? Rev. Sci. Eau 18: 307-330.

Göbel, A.; Thomsen, A., McArdell, C.S., Joss, A. \& Giger, W. 2005. Occurrence and sorption behavior of sulfonamides, macrolides, and trimethoprim in activated sludge treatment. Environ. Sci. Technol. 39: 3981-3989.

Gomez, M.J.; Petrovic, M. Fernandez-Alba, A.R. \& Barcelo, D. 2006. Determination of pharmaceuticals of various therapeutic classes by solid-phase extraction and liquid chromatography - tandem mass spectrometry analysis in hospital effluent wastewaters. J. Chromatography A, 1114: 224-233.

Gross, B.; Montgomery-Brown, J., Naumann, A. \& Reinhard, M. 2004. Occurrence and fate of pharmaceuticals and alkylphenol ethoxylate metabolites in an effluent-dominated river and wetland. Environ. Toxicol. Chem. 23: 2074-2083.

Halling-Sørensen, B.; Nielsen, N., Lanzky, P.F., Ingerslev, F.,Hansen, L., Lützhoft, H.C. \& Jørgensen, S.E. 1998. Occurrence, fate and effects of pharmaceutical substances in the environment - a review. Chemosphere 36: 357-394.

Halling-Sørensen, B.; Lützhoft, H.C., Andersen, H.R., \& Ingerslev, F. 2000. Environmental risk assessment of antibiotics: comparison of mecillinam, trimethoprim and ciprofloxacin. J. Antimicrobial Chemotherapy 46: 53-58.

Han, G.H.; Hur, H.G. \& Kim, S.D. 2006. Ecotoxicological risk of pharmaceuticals from wastewater treatment plants in Korea: occurrence and toxicity to Daphnia magna. Environ. Toxicol. Chem. 25: 265-271.

Hansen, H.C. 1999. Treatment of chronic pain with antiepileptic drugs : a new era. South Med. J. 92: 642-649.

Heberer, T. \& Feldmann, D. 2004. Removal of pharmaceutical residues from contaminated raw water sources by membrane filtration. In: Kümmerer, K. (ed.) Pharmaceuticals in the environment: sources, fate, effects and risks. Springer Berlin, 391$410 \mathrm{pp}$.

Hernando, M.D.; Mezcua, M., Fernandez-Alba, A.R. \& Barcelo, D. 2006. Environmental risk assessment of pharmaceutical residues in wastewater effluents, surface waters and sediments. Talanta 69 :334-342.

Hirsch, R.; Ternes, T., Heberer, K. \& Kratz, K.-L. 1999. Occurrence of antibiotics in the aquatic environment. Sci. Total Environ. 225: 109-118.

Holcombe, G.W.; Phipps, G.L., Sulaiman, A.H. \& Hoffman, A.D. 1987. Simultaneous multiple species testing: acute toxicity of 13 chemiclas to 12 diverse freshwater amphibian, fish, and invertebrate families. Arch. Environ. Contam. Toxicol. 16: 697-710.

Holten-Lützhoft, H.C.; Halling-Sorensen, B. \& Jorgensen, S.E. 1999. Algal toxicity of antibacterial agents applied in Danish fish farming. Arch. Environ. Toxicol. 36: 1-6.

Hua, W.Y.; Bennett, E.R., Xui-Sheng, M., Metcalfe, C. \& Letcher, R.J. 2006. Seasonality effects on pharmaceuticals and $S$-triazine herbicides in wastewater effluent and surface water from the Canadian side of the upper Detroit River. Environ. Toxicol. Chem. 25: 2356-2365.

IARC (International Agency for Research on Cancer), 1991. Coffee, tea, mate, methylxanthines and methlyglyoxal. World Health Organization, Lyon, France, Vol. 51, 513 pp.

Isidori, M.; Lavorgna, M., Nardelli, A., Parrella, A., Previtera, L. \& Rubino, M. 2005. Ecotoxicity of naproxen and its phototransformation products. Sci. Total Environ. 348: 93-101.

Jasim, S.Y.; Irabelli, A., Yang, P., Ahmed, S. \& Schweitzer, L. 2006. Presence of pharmaceuticals and pesticides in Detroit River water and the effect of ozone on removal. Ozone Sci. Engineering 28: 415-423.

Jjemba, P.K. 2006. Excretion and ecotoxicity of pharmaceutical and personal care products in the environment. Ecotox. Environ. Safety 63: 113-130.

Jones, O.A.H.; Voulvoulis, N. \& Lester, J.N. 2001. Human pharmaceuticals in the aquatic environment : a review. Environ. Technol. 22: 1383-1394.

Jones, O.A.H. ; Voulvoulis, N. \& Lester, J.N. 2002. Aquatic environmental assessment of the top 25 
English prescription pharmaceuticals. Water Res. 36: 5013-5022.

Jos, A.; Repetto, G., Rios, J.C., Hazen, M.J., Molero, M.L., del Peso, A., Salguero, M., Fernandez-Freire, Perez-Martin, J.M. \& Camean, A. 2003. Ecotoxicological evaluation of carbamazepine using six different model systems with eighteen endpoints. Toxicol. In vitro 17: 525-532.

Kasim, N.A.; Whitehouse, M., Ramachandran, C., ermejo, M., Lennernas, H., Hussain, A.S., Junginger, H.E., Stavchansky, S.A., Midha, K.K., Shah, V.P. \& Amidon, G.L. 2004. Molecular properties of WHO essential drugs and provisional biopharmaceutical classification. Mol. Pharmaceut. 1: 85-96.

Knoll, 1995. BASF Pharma safety data sheet: Ibuprofen. Issue/revision date 6/4/95. Knoll Pharmaceuticals. Nottingham, England.

Kolpin, D.W.; Furlong, E.T., Meyer, M.T., Thurman, E.M., Zaugg, S.D., Barber, L.B. \& Buxton, H.T. 2002. Pharmaceuticals, hormones, and other organic wastewater contaminants in U.S. streams, 19992000: a national reconnaissance. Environ. Sci. Technol. 36: 1202-1211.

Kummerer, K. 2001. Drugs in the environment: emission of drugs, diagnostic aids and disinfectants into wastewater by hospitals in relation to other sources - a review. Chemosphere 45: 957-969.

la Farré, M.; Ferrer, I., Ginebreda, A., Figueras, M., Olivella, L., Tirapu, L., Vilanova, M. \& Barcelo, D. 2001. Determination of drugs in surface water and wastewater samples by liquid chromatography-mass spectrometry: methods and preliminary results including toxicity studies with Vibrio fischeri. J. Chromatogr. A 938: 187-197.

Legault, R.; Blaise, C., Trottier, S. \& White, P. 1996. Detecting the genotoxic activity of industrial effluents with the SOS Chromotest microtitration procedure. Environ. Toxicol. Water Qual. 11: 151-165

Lilius, H.; Isomaa, B. \& Holström, T. 1994. A comparison of the toxicity of 50 reference chemicals to freshly isolated rainbow trout hepatocytes and Daphnia magna. Aquat. Toxicol. 30: 47-60.

Metcalfe, C.D.; Miao, X.-S., Koenig, B.G. \& Struger, J. 2003a. Distribution of acidic and neutral drugs in surface waters near sewage treatment plants in the lower Great lakes, Canada. Environ. Toxicol. Chem. 22 : 2881-2889.

Metcalfe, C.D.; Koenig, B.G., Bennie, D., Servos, M., Ternes, T.A. \& Hirsch, R. 2003b. Occurrence of neutral and acidic drugs in the effluents of Canadian sewage treatment plants. Environ. Toxicol. Chem. 22: $2872-2880$.

Metcalfe, C. ; Miao, X.S., Hua, W., Letcher, R. \& Servos, M. 2004. Pharmaceuticals in the Canadian environment. In: Kummerer, K. (ed.), Pharmaceuticals in the Environment-Sources, fate, effects and risks Springer, Berlin, 67-90 pp.

Miao, X.S. ; Bishay, F., Chen, M. \& Metclafe, C.D. 2004. Occurrence of antimicrobials in the final effluents of wastewater treatment plants in Canada. Environ. Sci. Technol. 38: 3533-3541.

Mimeault, C. ; Woodhouse, A.J., Miao, X.S., Metcalfe, C.D., Moon, T.W. \& Trudeau, V.L. 2005. The human lipid regulator, gemfibrozil, bioconcentrates and reduces testosterone in the goldfish, Carassius auratus. Aquat. Toxicol. 73: 44-54.

Nalecz-Jawecki, G. \& Persoone, G. 2006. Toxicity of selected pharmaceuticals to the anostracan crustacean Thamnocephalus platyurus Comparison of sublethal and lethal effect levels with the $1 \mathrm{~h}$ Rapidtoxkit and the 24h Thamnotoxkit microbiotests. Environ. Sci. Pollut. Res. 13: : 2227.

Ogunseitan, O.A., 1996. Removal of caffeine in sewage by Pseudomonas putida: Implications for water pollution index. World J. Microbiol. Biotechnol. 12: 251-256.

Pascoe, D.; Karntanut, W. \& Müller, C.T. 2003. Do pharmaceuticals affect freshwater invertebrates? A study with the cnidarian Hydra vulgaris. Chemosphere 51:521-528.

Paxéus, N. 2004. Removal of selected non steroidal anti-inflammatory drugs (NSAIDs), gemfibrozil, carbamazepine, â-blockers, trimethoprim and triclosan in conventional wastewater treatment plants in five EU countries and their discharge to the aquatic environment. Water Sci. Technol. 50: 253-260.

Pedersen, J.A.; Soliman, M. \& Suffet, H. 2005. Human pharmaceuticals, hormones, and personal care product ingredients in runoff from agricultural fields irrigated with treated wastewater. J. Agric. Food Chem. 53: 1625-1632.

Perez, S.; Eichhorn, P. \& Aga, D. 2005. Evaluating the biodegradability of sulfamethazine, sulfamethoxazole, sulfathiazole, and trimethoprim at different stages of sewage treatment. Environ. Toxicol. Chem. 24: 1361-1367.

Pham, T.T. \& Proulx S. 1997. PCBs and PAHs in the Montreal urban community (Québec, Canada) wastewater treatment plant and in the effluent plume in the St-Lawrence River. Wat. Res. 31: 1887-1896.

Pomati, F.; Netting, A., Calamari, D. \& Neilan, B. 2004. Effects of erythromycin, tetracycline and ibuprofen on the growth of Synechocystis sp. and Lemna minor. Aquat. Toxicol. 67: 387-396.

Pro, J.; Ortiz, J.A., Boleas, S., Fernandez, C., Carbonell, G. \& Tarazona, J.V. 2003. Effect assessment of antimicrobial pharmaceuticals on the aquatic plant 
Lemna minor. Bull. Environ. Contam. Toxicol. 70: 290-295.

Quémerais, B.; Cossa, D., Rondeau, B., Pham, T.T. \& Fortin, B. 1998. Mercury distribution in relation to iron and manganese in waters of the St-lawrence River. Sci. Total Environ. 213: 193-201.

Quinn, B.; Gagné, F. \& Blaise, C. 2004. Oxidative metabolism in Hydra attenuata exposed to carbamazepine. Fresenius Environmental Bulletin 13: 783-788.

Richardson, M. L. \& Brown, J.M. 1985. The fate of pharmaceutical chemicals in the aquatic environment. J. Pharmacol. 37: 1-12.

Roberts, P.H. \& Thomas, K.V. 2006. The occurrence of selected pharmaceuticals in wastewater effluent and surface waters of the lower Tyne catchment. Sci. Total Environ. 356: 143-153.

Rusom, C.L.; Bradbury, S.P., Broderius, S.J., Hammermeister, D.E. \& Drummond, R.A. 1997. Predicting modes of toxic action from chemical structure: acute toxicity in the fathead minnow (Pimephales promelas). Environ. Toxicol. Chem. 16: 948-967.

Sanderson, H.; Johnson, D.J., Reitsma, T., Brain, R.A. \& Solomon, K.R. 2004. Ranking and prioritization of environmental risks of pharmaceuticals in surface water. Regulat. Toxicol. Pharmacol 39: 158-183.

Schowanek, D. \& Webb, S. 2000. Examples of exposure assessment simulation for pharmaceuticals in river basins with the GREAT-ER 1.0 system. Proceedings KVIV Seminar "Pharmaceuticals in the environment" March 9, 2000, Brussels.

Servos, M.; Bennie, D.T., Starodub, M.E., Orr, J.C. 2002. Pharmaceuticals and personnal care products in the environment: a summary of published literature. National Water Research Institute, Contribution No. 02-309. Environment Canada, $52 \mathrm{pp}$.

Siegener, R. \& Chen. R.F. 2002. Caffeine in Boston Harbor seawater. Mar. Pollut. Bull. 44: 383-387.

Smith, S.B.; Savino, J.F. \& Blouin, M.A. 1988. Acute toxicity to Daphnia pulex of six classes of chemical compounds potentially hazardous to Great Lakes aquatic biota. J. Great Lakes Res. 14: 394-404.

Stackelberg, P.E.; Furlong, E.T., Meyer, M.T., Zaugg, S.D., Henderson, A.K. \& Reissman, D.B. 2004. Persistence of pharmaceutical compounds and other organic wastewater contaminants in a conventional drinking-water treatment plant. Sci. Total Environ. 329: 99-113.

Stumpf, M.; Ternes, T.A., Wilken, R.-D., Rodrigues, S.V. \& Baumenn, W. 1999. Polar drug residues in sewage and natural waters in the state of Rio de Janeiro, Brazil. Sci. Total Environ. 225: 135-141.
Ternes, T.A. 1998. Occurrence of drugs in German sewage treatment plants and rivers. Water Res. 32: 3245-3260.

Thomulka, K.W.; McGee, D.J. \& Lange, J.H. 1993. Detection of biohazardous materials in water by measuring bioluminescence reduction with the marine organism Vibrio Harveyi. J. Environ. Sci. Health A28: 2153-2166.

Tixier, C.; Singer, H.P., Oellers, S. \& Muller, S.R. 2003. Occurrence and fate of carbamazepine, clofibric acid, diclofenac, ibuprofen, ketoprofen, and naproxen in surface waters. Environ. Sci. Technol. 37 : 10611068.

Vane, J.R. \& Botting, R.M. 1998. Mechanism of action of nonsteroidal anti-inflammatory drugs. Am. J. Med. 104(3A): 2S-8S.

Webb, S.F. 2004. A data-based perspective on the environmental risk assessment of human pharmaceuticals I-Collation of available ecotoxicity data. In: Kummerer, K. (ed.), Pharmaceuticals in the Environment - Sources, fate, effects and risks Springer, Berlin, 317-343 pp.

White, P.; Rasmussen, J. \& Blaise, C. 1996. Comparing the presence, potency and potential hazard of organic compounds extracted from a broad range of industrial effluents. Environ. Molec. Mutagen. 27: 116-139.

Winston, G.W.; Moore, M.N., Kirchin, M.A. \& Soverchia, C. 1996. Production of reactive oxygen species by hemocytes from the marine mussel, Mytilus edulis: lysosomal localization and effects of xenobiotics. Comp. Biochem. Physiol. 113C: 221-229.

Wollenberger, L.; Halling-Sorensen, B. \& Kusk, K.O. 2000. Acute and chronic toxicity of veterinary antibiotics to $D$. magna. Chemosphere 40:723-730.

Zhu, H.; Banneberg, G.L., Moldeaus, P., Shertzer, H.G. 1994. Oxidation pathways for the intracellular probe 2',7'-dichlorofluorescin. Arch. Toxicol. 68: 582-587.

Zuccato, E.; Bagnati, R., Fioretti, F., Natangelo, M., Calamari, D. \& Fanelli, R. 2001. In: K. Kümmerer (ed.) Pharmaceuticals in the environment (first edition). Springer-Verlag, Berlin, 19-27 pp.

Zurita, J.L.; Repetto, G., Jos, A., Salguero, M., LopezArtiguez, M. \& Camean, A.M. 2006. Toxicological effects of the lipid regulator gemfibrozil in four aquatic systems. Aquat. Toxicol. 81: 106-115.

Zwiener, C. \& Frimmel, F. 2004. Residues of clofibric acid, ibuprofen and diclofenac in the aquatic environment and their elimination in sewage treatment and drinking water production. In: $\mathrm{K}$. Kümmerer (ed.) Pharmaceuticals in the environment: sources, fate, effects and risks (second edition). Springer-Verlag Berlin, 121-137 pp. 